\title{
Study of Exponential Growth Constants of Directed Heteropolygonal Archimedean Lattices
}

\author{
Shu-Chiuan Chang ${ }^{a}$ and Robert Shrock $^{b}$ \\ (a) Department of Physics, National Cheng Kung University, Tainan 70101, Taiwan and \\ (b) C. N. Yang Institute for Theoretical Physics and Department of Physics and Astronomy \\ Stony Brook University, Stony Brook, NY 11794, USA
}

We infer upper and lower bounds on the exponential growth constants $\alpha(\Lambda), \alpha_{0}(\Lambda)$, and $\beta(\Lambda)$ describing the large- $n$ behavior of, respectively, the number of acyclic orientations, acyclic orientations with a unique source vertex, and totally cyclic orientations of arrows on bonds of several $n$-vertex heteropolygonal Archimedean lattices $\Lambda$. These are, to our knowledge, the best bounds on these growth constants. The inferred upper and lower bounds on the growth constants are quite close to each other, which enables us to derive rather accurate values for the actual exponential growth constants. Combining our new results for heteropolygonal Archimedean lattices with our recent results for homopolygonal Archimedean lattices, we show that the exponential growth constants $\alpha(\Lambda), \alpha_{0}(\Lambda)$, and $\beta(\Lambda)$ on these lattices are monotonically increasing functions of the lattice coordination number. Comparisons are made with the corresponding growth constants for spanning trees on these lattices. Our findings provide further support for the Merino-Welsh and Conde-Merino conjectures. 


\section{INTRODUCTION}

In this paper we continue our study of the exponential growth constants $\alpha(\Lambda), \alpha_{0}(\Lambda)$, and $\beta(\Lambda)$ describing the large- $n$ behavior of, respectively, the number of acyclic orientations, acyclic orientations with a unique source vertex, and totally cyclic orientations of arrows on bonds of $n$-vertex Archimedean lattices $\Lambda$. In Ref. [1] we inferred upper and lower bounds on these exponential growth constants for several lattices, including the three homopolygonal Archimedean lattices: square $(s q)$, triangular (tri), and honeycomb $(h c)$. For each growth constant and lattice, our upper and lower bounds in [1] were quite close to each other, which allowed us to obtain reasonably precise values for the actual exponential growth constants. We also presented exact values for $\alpha(t r i), \alpha_{0}(t r i)$, and $\beta(h c)$. In the present paper we extend this analysis to heteropolygonal Archimedean lattices.

We begin with some definitions and background. We refer the reader to our previous paper [1] for more details and to [2] for general discussions of mathematical graph theory. A graph $G=(V, E)$ is defined by its vertex and edge sets $V$ and $E$. We denote $n(G)=|V|$, $e(G)=|E|, f c(G)$, and $k(G)$ as the number of vertices (=sites), edges (= bonds), faces, and connected components of $G$, respectively. We will focus on planar lattice graphs with various boundary conditions in the longitudinal and transverse directions, taken as the $x$ and $y$ directions, respectively. The degree of a vertex in a graph is the number of edges that connect to it. A graph whose vertices have the same degree $\Delta$ is termed a $\Delta$-regular graph. An Archimedean lattice is a uniform tiling of the plane with one or more types of regular polygons, such that all vertices are equivalent [3]. A graph that is a finite section of an Archimedean lattice with doubly periodic boundary conditions is a $\Delta$-regular graph, and we will use this term also in the limit $n(\Lambda) \rightarrow \infty$ (where it is synonymous with the lattice coordination number), denoting it as $\Delta(\Lambda)$. If an Archimedean lattice is comprised of only a single type of regular polygon, it is termed homopolygonal, while if it is comprised of two or more different types of regular polygons, it is termed heteropolygonal. Owing to the equivalence of all vertices of an Archimedean lattice $\Lambda$, it may be defined by the ordered sequence of regular polygons that one traverses in a circuit around any vertex:

$$
\Lambda=\left(\prod_{i} p_{i}^{a_{i}}\right)
$$

where the $i$ 'th polygon has $p_{i}$ sides and appears $a_{i}$ times contiguously in the sequence (it can also occur non-contiguously). The total number of occurrences of the polygon $p_{i}$ in the above sequence is denoted as $a_{i, s}$. The number of polygons of type $p_{i}$ per vertex is $\nu_{p_{i}}=a_{i, s} / p_{i}$. There are eleven Archimedean lattices, listed in Eqs. (A1)-(A4) in the Appendix. Of these, three are homopolygonal, namely $\left(4^{4}\right)$ (square), $\left(3^{6}\right)$ (triangular), and $\left(6^{3}\right)$ (honeycomb), and 
the rest are heteropolygonal.

Given a graph $G$, we assign an arrow to each edge of $G$, thus defining a directed graph (also called a digraph), $D(G)$. Since the arrow on each edge has two possible orientations, there are $N_{e o}(G)=2^{e(G)}$ possible orientations of these arrows on edges of $G$ (where the subscript eo stands for "edge orientations"). An interesting and fundamental question in graph theory concerns the numbers of certain subsets of the $N_{e o}(G)$ edge (arrow) orientations and how these numbers grow as $n(G) \rightarrow \infty$. Here we focus on three classes of arrow orientations. We restrict our analysis of these subsets of arrow orientations to connected graphs $G$, so $k(G)=1$; this does not entail any loss of generality. A directed cycle on a directed graph $D(G)$ is defined as a set of arrows on edges forming a cycle (circuit) such that, as one traverses the cycle in a given direction, all of the arrows point in the direction of motion. An acyclic orientation of the arrows on edges of $D(G)$ is one in which there are no directed cycles. We denote $a(G)$ as the number of acyclic orientations on the graph $G$. Now consider a given vertex in $G$. One may enumerate the number of acyclic orientations on $G$ for which this, and only this, vertex is a source vertex, i.e. has outgoing arrows on all edges connected to it. This number is actually independent of the choice of the vertex, and is denoted $a_{0}(G)$. Among the various orientations of the arrows on edges of $G$, some have the property that the arrow on each edge is a member of a directed cycle. These are called totally cyclic (edge) orientations. The number of these, denoted as $b(G)$, constitutes a third basic quantity of interest. We restrict our analysis to graphs without loops (i.e., edges that connect a vertex to itself), since if a graph $G$ has a loop, then $a(G)$ and $a_{0}(G)$ both vanish identically. In order to have a minimal measure of totally cyclic orientations, we also restrict our analysis to graphs without multiple edges, since one can increase $b(G)$ arbitrarily by replacing single edges by multiple edges in a given graph.

The numbers $a(G)$ and $a_{0}(G)$ can be calculated from a knowledge of the chromatic polynomial $P(G, q)$ of $G$, which enumerates the number of assignments of $q$ colors to the vertices of $G$ satisfying the condition that the colors on any two adjacent vertices (i.e., vertices connected by an edge) are different [4]. Such a color assignment is called a proper $q$-coloring of (the vertices of) $G$. The minimum value of $q$ for which one can perform a proper $q$-coloring of $G$ is the chromatic number of $G$, denoted $\chi(G)$. The chromatic polynomial always has an overall factor of $q$, so that one can define a reduced polynomial $P_{r}(G, q) \equiv q^{-1} P(G, q)$. Although the chromatic polynomial $P(G, q)$ enumerates proper $q$-colorings for positive integer values of $q$, it is also well-defined for other values of $q$. Specifically, $a(G)=(-1)^{n(G)} P(G,-1)$ [5] and $a_{0}(G)=(-1)^{n(G)-1} P_{r}(G, 0)$ [6]. As discussed in the Appendix, the chromatic polynomial is a special case of an important (two-variable) graph-theoretic function, namely the Tutte polynomial, $T(G, x, y)$ [7]-[10], [2], and equivalent expressions for $a(G)$ and $a_{0}(G)$ are 
$a(G)=T(G, 2,0)$ [5] and $a_{0}(G)=T(G, 1,0)[6,11]$. Some previous studies of acyclic orientations on square-lattice graphs include [12]-[14]. The number $b(G)$ can be determined in terms of the flow polynomial $F(G, q)$ evaluated at $q=-1$ or equivalently in terms of the Tutte polynomial as $b(G)=T(G, 0,2)[15,16]$.

For a wide class of families of lattice strip graphs $G$ of a given width and arbitrarily great length, with certain longitudinal and transverse boundary conditions, the quantities $a(G), a_{0}$, and $b(G)$ grow exponentially rapidly as a function of $n(G) \equiv n$ as $n \rightarrow \infty$. Let $\{G\}$ denote the formal limit of a given family of lattice strip graphs as $n \rightarrow \infty$. One thus defines exponential growth constants (EGCs) that describe the asymptotic growth of these quantities as $n \rightarrow \infty$ :

$$
\begin{aligned}
\alpha(\{G\}) & =\lim _{n \rightarrow \infty}[a(G)]^{1 / n}, \\
\alpha_{0}(\{G\}) & =\lim _{n \rightarrow \infty}\left[a_{0}(G)\right]^{1 / n},
\end{aligned}
$$

and

$$
\beta(\{G\})=\lim _{n \rightarrow \infty}[b(G)]^{1 / n} .
$$

A recursive family of graphs is a family such that the $(m+1)^{\prime}$ th member of the family can be obtained from the $m$ 'th member by adding a copy of a given subgraph [17]. An example is a family of lattice strips with a fixed width $L_{y}$ and variable length $m$, together with some specified set of longitudinal and transverse boundary conditions. For a recursive family of graphs, in particular, a family of strips of some lattice $\Lambda$, the Tutte polynomial $T(G, x, y)$, or equivalently, the Potts model partition function $Z(G, q, v)$ (see Eqs. (A21), (A23) and (A24) in the Appendix), and hence also the chromatic polynomial, can be written as a sum of $m$ 'th powers of certain functions, the set of which is generically denoted $\{\lambda\}$, that depend on the type of lattice $\Lambda$, the strip width $L_{y}$, and the boundary conditions, but not on the length, $m$. In the infinite-length limit $m \rightarrow \infty$, the $\lambda$ function with the largest magnitude dominates the sum. Hence, when calculating the exponential growth constants in the limit of infinitely long finite-width strips, it is only necessary to calculate the dominant $\lambda$ function. From our previous calculations of chromatic and Tutte/Potts polynomials for a variety of lattice strip graphs, we know what these dominant $\lambda$ functions are at the values $(q, v)=(-1,-1)$, $(0,-1)$, and $(-1,1)$ (or equivalently, in terms of Tutte variables, $(x, y)=(2,0),(1,0)$, and $(0,2))$ needed to evaluate $\alpha(\{G\}), \alpha_{0}(\{G\})$, and $\beta(\{G\})$, respectively.

Because the Tutte polynomial $T(G, x, y)$ is equivalent to the partition function of the $q$-state Potts model in statistical mechanics, $Z(G, q, v)$, with $x=1+(q / v)$ and $y=v+1$, the quantities $a(G), a_{0}(G)$, and $b(G)$ and the associated exponential growth constants $\alpha(\{G\}), \alpha_{0}(\{G\})$, and $\beta(\{G\})$ have interesting connections with physical quantities, albeit at different values of $q$ than one studies in physical situations. Specifically, $\alpha(\{G\})=$ 
$|W(\{G\},-1)|, \alpha_{0}(\{G\})=|W(\{G\}, 0)|$, and $\beta(\{G\})=e^{|f(\{G\},-1,1)|}$, where $W(\{G\}, q)$ is the zero-temperature degeneracy per vertex of the Potts antiferromagnet and $f(\{G\}, q, v)$ is the dimensionless free energy per vertex of the Potts model, defined in Eqs. (A22) and (A25). It will also be of interest to compare these numbers with the exponential growth constant $\tau(\{G\})$ describing the asymptotic growth of the number of spanning trees on $G$ as $n(G) \rightarrow \infty$.

For a $\Delta$-regular graph $G, N_{e o}(G)$, the number of edge orientations, is given by the formula above with $e(G)=\Delta(G) n(G) / 2$, i.e., $N_{e o}=2^{\Delta(G) n(G) / 2}$. We will also investigate some properties of lattice strips of (heteropolygonal) Archimedean lattices and duals of Archimedean lattices, some of which are not $\Delta$-regular graphs. For this purpose, we define an effective vertex degree as $\Delta_{\text {eff }}(G)=2 e(G) / n(G)$ and $\Delta_{e f f}(\{G\})=\lim _{n \rightarrow \infty} 2 e(G) / n(G)$, as in [18]. This leads naturally to the definition of an exponential growth constant for $N_{e o}$. For a $\Delta$ regular graph, this is $\epsilon(\{G\}) \equiv \lim _{n(G) \rightarrow \infty}\left[N_{e o}(G)\right]^{1 / n(G)}=2^{\Delta(\{G\}) / 2}$ and for a general graph, it is $\epsilon(\{G\})=2^{\Delta_{e f f}(\{G\}) / 2}$.

This paper is organized as follows. In Section II we illustrate the calculation of exponential growth constants with a specific example of a strip of a heteropolygonal lattice. In Section III we briefly review our method of obtaining upper and lower bounds on these exponential growth constants. Our resulting bounds are presented in Section IV] We discuss an interesting connection with bounds on the zero-temperature degeneracy per vertex of the Potts antiferromagnet in V. In Section VI we present inferred upper bounds on exponential growth constants for duals of Archimedean lattices. Our results are given in Tables I.XXVII. A comparative discussion is given in Section VII, and our conclusions are stated in Section VIII. Some useful results from graph theory are included in an Appendix.

\section{ILLUSTRATIVE CALCULATIONS FOR KAGOMÉ STRIP}

Here we give a brief illustration of how exponential growth constants can be calculated for strips of one type of heteropolygonal lattice, namely the $(3 \cdot 6 \cdot 3 \cdot 6)$ lattice. This is commonly called the kagomé lattice, and we will use the abbreviation kag for it. We take the longitudinal and transverse directions to be the $x$ and $y$ directions, respectively, and denote the boundary conditions as $\left(B C_{y}, B C_{x}\right)$. (These symbols $x$ and $y$ should not be confused with the variables $x$ and $y$ in the Tutte polynomial $T(G, x, y)$ in Eq. (A23); the context will always make clear the distinction.) The boundary conditions $\left(B C_{y}, B C_{x}\right)$ are labelled as $(\mathrm{F}, \mathrm{F})=$ free, $(\mathrm{F}, \mathrm{P})=$ cyclic, $(\mathrm{P}, \mathrm{F})=$ cylindrical, and $(\mathrm{P}, \mathrm{P})=$ toroidal. Our bounds for $\alpha(\{G\})$, and $\alpha_{0}(\{G\})$ are independent of $B C_{x}$ but depend on $B C_{y}$, while the 
bounds for $\beta(\{G\})$ depend on both $B C_{x}$ and $B C_{y}$.

For definiteness, in this section we consider kagomé strips with (F,P), i.e., cyclic, boundary conditions. The repeating subgraph in the first such strip of this type consists of a hexagon and two adjacent triangles, say the upper and lower left triangles (see Fig. 1(f) of [19]). This strip graph is denoted $\left\{\right.$ kagmin $\left._{m}, c y c\right\}$, where the abbreviation kagmin stands for "kagomé strip of minimal width", and cyc stands for cyclic. A strip of this type, with length $m$ of these subgraphs, has $n=5 m$ vertices and $8 m$ edges. It contains vertices with degree 3 and 4 , and has an effective vertex degree $\Delta_{e f f}=16 / 5=3.20$. The chromatic polynomial for this cyclic strip was given in [20, 21] in terms of a generating function. In an equivalent form, we write it as

$$
\begin{aligned}
P\left(\text { kagmin }_{m}, \text { cyc }, q\right) & =\left(\lambda_{\text {kag }, 0,+}\right)^{m}+\left(\lambda_{\text {kag }, 0,-}\right)^{m}+(q-1)\left[\left(\lambda_{\text {kag }, 1,+}\right)^{m}+\left(\lambda_{\text {kag }, 1,-}\right)^{m}+\left(\lambda_{\text {kag }, 1,3}\right)^{m}\right] \\
& +\left(q^{2}-3 q+1\right)\left(\lambda_{\text {kag }, 2}\right)^{m}
\end{aligned}
$$

where

$$
\begin{gathered}
\lambda_{k a g, 0, \pm}=\frac{(q-2)}{2}\left(T_{k a g 0} \pm \sqrt{R_{k a g 0}}\right) \\
T_{k a g 0}=q^{4}-6 q^{3}+14 q^{2}-16 q+10 \\
R_{k a g 0}=q^{8}-12 q^{7}+64 q^{6}-200 q^{5}+404 q^{4}-548 q^{3}+500 q^{2}-292 q+92 \\
\lambda_{k a g, 1, \pm}=\frac{1}{2}\left(T_{k a g 1} \pm \sqrt{R_{k a g 1}}\right) \\
T_{k a g 1}=q^{3}-7 q^{2}+19 q-20 \\
R_{k a g 1}=q^{6}-14 q^{5}+83 q^{4}-278 q^{3}+569 q^{2}-680 q+368 \\
\lambda_{k a g, 1,3}=(q-1)(q-2)^{2}
\end{gathered}
$$

and

$$
\lambda_{k a g, 2}=q-4 .
$$

Evaluating $P\left(\right.$ kagmin $\left._{m}, c y c, q\right)$ at $q=-1$, we obtain

$$
\begin{aligned}
a_{\left(\text {kagmin }_{m}, \text { cyc }\right)} & =\left[\frac{3(47+\sqrt{2113})}{2}\right]^{m}+\left[\frac{3(47-\sqrt{2113})}{2}\right]^{m} \\
& -2\left[\left(\frac{47+\sqrt{1993}}{2}\right)^{m}+\left(\frac{47-\sqrt{1993}}{2}\right)^{m}+(18)^{m}\right]+5^{m+1} .
\end{aligned}
$$


Taking the $m \rightarrow \infty$ limit yields the result

$$
\alpha\left(\left\{\text { kagmin }_{2 \times \infty}\right\}\right)=\left[\frac{3(47+\sqrt{2113})}{2}\right]^{1 / 5}=2.684630 .
$$

Similarly, calculating $a_{0}\left(m_{k a g_{m}}, c y c\right)$ and taking $m \rightarrow \infty$, we get

$$
\alpha_{0}\left(\left\{\operatorname{kagmin}_{2 \times \infty}\right\}\right)=[2(5+\sqrt{23})]^{1 / 5}=1.813069
$$

A second type of cyclic strip graph of the kagomé lattice can be constructed from the first by adjoining triangles to each of the edges of hexagons on one side of the strip, say the upper side, so that the basic subgraph that repeats $m$ times is a hexagon with three adjacent triangles, on the upper and lower left of a given hexagon and above it. We denote this strip graph as $\left\{k a g_{m}, c y c\right\}$. It has $n=6 m$ vertices and $10 m$ edges. It contains vertices with degrees 2,3 , and 4 , and has an effective $\Delta_{e f f}=10 / 3=3.33$. Knowing $P\left(\right.$ kagmin $\left._{m}, c y c, q\right)$, an elementary calculation yields

$$
P\left(k_{m}, c y c, q\right)=(q-2)^{m} P\left(\text { kagmin }_{m}, c y c, q\right) .
$$

Hence,

$$
\alpha\left(k a g_{2 \times \infty}\right)=3^{1 / 3}\left(\frac{47+\sqrt{2113}}{2}\right)^{1 / 6}=2.734789
$$

and

$$
\alpha_{0}\left(\operatorname{kag}_{2 \times \infty}\right)=2^{1 / 3}(5+\sqrt{23})^{1 / 6}=1.842964 .
$$

The values in Eqs. (2.14) and (2.15) are listed in Table VII More generally, the results for infinite-length finite-width strips of the kagomé lattice presented in Table VII use a strip comprised of $L_{y}-1$ layers of kagmin glued to one layer of $k a g$, i.e., they have triangles protruding on one side, say the upper one, but a "flat" lower side.

A third type of cyclic strip graph of the kagomé lattice can be constructed from the first by adjoining triangles to each of the edges of hexagons on both the upper and the lower sides, so that the basic subgraph that repeats $m$ times is a hexagon with four adjacent triangles, on the upper and lower left of a given hexagon and above and below it. We denote this strip graph as kagt $_{m}$, where the $t$ in kagt refers to the additional triangle subgraphs. It has $n=7 m$ vertices, $12 m$ edges and an effective vertex degree $\Delta_{e f f}=24 / 7=3.429$. Another elementary calculation yields

$$
P\left(k_{a g t}, c y c, q\right)=(q-2)^{m} P\left(k_{a g}, c y c, q\right)=(q-2)^{2 m} P\left(k_{a g m i n}, c y c, q\right) .
$$

Hence,

$$
\alpha\left(\{\text { kagt }\}_{2 \times \infty}\right)=3^{3 / 7}\left(\frac{47+\sqrt{2113}}{2}\right)^{1 / 7}=2.771190
$$


and

$$
\alpha_{0}\left(\{k a g t\}_{2 \times \infty}\right)=2^{3 / 7}(5+\sqrt{23})^{1 / 7}=1.864619 .
$$

The fact that these exponential growth constants increase as the width of the strip increases is consistent with the inference that these provide lower bounds on $\alpha(k a g)$ and $\alpha_{0}(\mathrm{kag})$. This is the same type of behavior that we showed for homopolygonal lattice strips in [1, 14]. Similar illustrative calculations can be given for other strips.

\section{METHODS FOR CALCULATION OF UPPER AND LOWER BOUNDS ON EXPONENTIAL GROWTH CONSTANTS}

In our previous studies [1, 14] we showed that the resultant values of $\xi\left(\Lambda,\left(L_{y}\right)_{F} \times \infty\right)$ and $\xi\left(\Lambda,\left(L_{y}\right)_{P} \times \infty\right)$ were monotonically increasing functions of $L_{y}$ for all of the widths $L_{y}$ of homopolygonal lattices considered, where $\xi$ denotes any of the exponential growth constants $\alpha, \alpha_{0}$, and $\beta$. Our results for heteropolygonal lattice strips exhibit the same monotonicity, providing further support for the inference that these quantities are lower bounds on the values of the respective exponential growth constants for the infinite lattices. Our results also provide further support for our earlier inference in [1, 14] that as $L_{y} \rightarrow \infty$, the values of $\xi\left(\Lambda,\left(L_{y}\right)_{F} \times \infty\right)$ and $\xi\left(\Lambda,\left(L_{y}\right)_{P} \times \infty\right)$ converge to the same unique value, denoted $\xi(\Lambda)$, which is independent of the longitudinal and transverse boundary conditions and thus characterizes the infinite lattice $\Lambda$. Since the strips with periodic transverse boundary conditions (cylindrical or toroidal) have no transverse boundary, the resulting values of the exponential growth constants on finite-width, infinite-length strips should approach the respective values for the infinite two-dimensional lattices more rapidly, and we do observe this for strips of heteropolygonal lattices, as we did earlier in [1, 14] for strips of homopolygonal lattices.

As in [1], as a quantitative measure the convergence of values of $\alpha\left(\Lambda,\left(L_{y}\right)_{B C_{y}} \times \infty\right)$ for consecutive values of strip width to a constant limiting value, we define the ratio

$$
R_{\alpha, \Lambda,\left(L_{y}+1\right) / L_{y}, B C_{y}} \equiv \frac{\alpha\left(\Lambda,\left(L_{y}+1\right)_{B C_{y}} \times \infty\right)}{\alpha\left(\Lambda,\left(L_{y}\right)_{B C_{y}} \times \infty\right)} .
$$

Just as was the case in [1] for homopolygonal lattice strips, we find that this ratio approaches close to 1 even for modest values of the strip widths. Our results for $\beta$ values in [1]

In [1] we showed that ratios of the $\lambda$ functions for successive strip widths provide an upper bound on the respective exponential growth constants. We refer the reader to [1] for this discussion. We proceed to present our results for the heteropolygonal Archimedean lattices that we study. 


\section{UPPER AND LOWER BOUNDS ON EXPONENTIAL GROWTH CONSTANTS ON HETEROPOLYGONAL ARCHIMEDEAN LATTICES}

In this section we present upper and lower bounds that we have inferred for $\alpha(\Lambda), \alpha_{0}(\Lambda)$, and $\beta(\Lambda)$ on the heteropolygonal Archimedean lattices $\Lambda$ that we study. We obtain these bounds via the calculations of infinite-length, finite-width strips of these lattices, using methods discussed in [1] and reviewed in Section [II.

\section{A. $\left(4 \cdot 8^{2}\right)$ Lattice}

We present our results for the $\left(4 \cdot 8^{2}\right)$ lattice in Tables [IVI] In these and later tables, because we utilize the entries with the highest values of the width $L_{y}$ of strips for our upper and lower bounds, we list these to slightly higher precision than the entries for smaller widths. As is evident from these tables and the others to be given below, we achieve very good precision in our upper and lower bounds with modest values of $L_{y}$ for the lattice strips. This was also true of our calculations on the homopolygonal Archimedean lattices in [1]. From these new results, we infer the following upper and lower bounds:

$$
\begin{aligned}
& 2.729704176<\alpha\left(\left(4 \cdot 8^{2}\right)\right)<2.730093140 \\
& 2.032649948<\alpha_{0}\left(\left(4 \cdot 8^{2}\right)\right)<2.077301063
\end{aligned}
$$

and

$$
2.080338691<\beta\left(\left(4 \cdot 8^{2}\right)\right)<2.107715225 \text {. }
$$

As was the case with our upper and lower bounds for the homopolygonal Archimedean lattices in [1], these bounds are quite close to each other, which enables us to infer approximate values of the exponential growth constants themselves. As a measure of this, for a general Archimedean lattice $\Lambda$, we define the fractional difference

$$
\frac{\xi_{u}(\Lambda)-\xi_{\ell}(\Lambda)}{\xi_{\text {ave }}(\Lambda)}
$$

where $\xi(\Lambda)$ is any of the growth constants, $\alpha(\Lambda), \alpha_{0}(\Lambda)$, or $\beta(\Lambda)$ and $\xi_{u}(\Lambda)$, and $\xi_{\ell}(\Lambda)$ are the corresponding upper $(u)$ and lower $(\ell)$ bounds. We further define the average value

$$
\xi_{\text {ave }}(\Lambda)=\frac{\xi_{\ell}(\Lambda)+\xi_{u}(\Lambda)}{2}
$$

For the $\left(4 \cdot 8^{2}\right)$ lattice, we have

$$
\frac{\alpha_{u}\left(\left(4 \cdot 8^{2}\right)\right)-\alpha_{\ell}\left(\left(4 \cdot 8^{2}\right)\right)}{\alpha_{\text {ave }}\left(\left(4 \cdot 8^{2}\right)\right)}=1.425 \times 10^{-4}
$$




$$
\frac{\alpha_{0, u}\left(\left(4 \cdot 8^{2}\right)\right)-\alpha_{0, \ell}\left(\left(4 \cdot 8^{2}\right)\right)}{\alpha_{0, \text { ave }}\left(\left(4 \cdot 8^{2}\right)\right)}=2.17 \times 10^{-2}
$$

and

$$
\frac{\beta_{u}\left(\left(4 \cdot 8^{2}\right)\right)-\beta_{\ell}\left(\left(4 \cdot 8^{2}\right)\right)}{\beta_{\text {ave }}\left(\left(4 \cdot 8^{2}\right)\right)}=1.31 \times 10^{-2} .
$$

The interval separating the average value of $\xi(\Lambda)$ from the upper and lower bounds is

$$
\delta_{\xi(\Lambda)} \equiv \xi_{u}(\Lambda)-\xi_{\text {ave }}(\Lambda)=\xi_{\text {ave }}(\Lambda)-\xi_{\ell}(\Lambda)
$$

The approximate $(a p)$ values of the exponential growth constants, denoted $\xi_{a p}(\Lambda)$ for $\xi=$ $\alpha, \alpha_{0}$, and $\beta$, are given by

$$
\xi_{a p}(\Lambda)=\xi_{\text {ave }}(\Lambda) \pm \delta_{\xi(\Lambda)}
$$

We calculate

$$
\begin{gathered}
\alpha_{a p}\left(\left(4 \cdot 8^{2}\right)\right)=2.72990 \pm 0.00019 \\
\alpha_{0, a p}\left(\left(4 \cdot 8^{2}\right)\right)=2.055 \pm 0.022
\end{gathered}
$$

and

$$
\beta_{a p}\left(\left(4 \cdot 8^{2}\right)\right)=2.094 \pm 0.014
$$

\section{B. $(3 \cdot 6 \cdot 3 \cdot 6)$ (Kagomé) Lattice}

We present our results for the $(3 \cdot 6 \cdot 3 \cdot 6)$ (kagomé) lattice in Tables VII XII. From these we infer the following upper and lower bounds:

$$
\begin{gathered}
3.249059070<\alpha(\mathrm{kag})<3.265737199 \\
2.481974714<\alpha_{0}(\mathrm{kag})<2.632503652
\end{gathered}
$$

and

$$
3.415032724<\beta(\mathrm{kag})<3.549454037 \text {. }
$$

The fractional differences $\left[\xi_{u}(k a g)-\xi_{\ell}(k a g)\right] / \xi_{\text {ave }}(k a g)$ are of order $10^{-2}$ for $\xi=\alpha, \alpha_{0}, \beta$. We compute

$$
\begin{gathered}
\alpha_{a p}(k a g)=3.2574 \pm 0.0083 \\
\alpha_{0, a p}(k a g)=2.557 \pm 0.075
\end{gathered}
$$

and

$$
\beta_{a p}(k a g)=3.482 \pm 0.067
$$




\section{C. $\left(3^{3} \cdot 4^{2}\right)$ Lattice}

We present our results for the $\left(3^{3} \cdot 4^{2}\right)$ lattice in Tables XIIT XVIII. For this lattice there are two different ways to choose the longitudinal direction for the strips. Referring to Fig. 1(a) in [22], we can choose either $L_{y}$ in the vertical direction and $L_{x}$ in the horizontal direction, or vice versa. We give results for both cases and use the most stringent ones (the largest lower bound and the smallest upper bound) for our results. We obtain

$$
\begin{aligned}
& 3.922582062<\alpha\left(\left(3^{3} \cdot 4^{2}\right)\right)<3.956121920 \\
& 3.142411228<\alpha_{0}\left(\left(3^{3} \cdot 4^{2}\right)\right)<3.298937504
\end{aligned}
$$

and

$$
5.262880165<\beta\left(\left(3^{3} \cdot 4^{2}\right)\right)<5.362606470 \text {. }
$$

The fractional differences $\left[\xi_{u}(\Lambda)-\xi_{\ell}(\Lambda)\right] / \xi_{\text {ave }}(\Lambda)$, where $\xi=\alpha, \alpha_{0}, \beta$, are of order $10^{-2}$ for this $\Lambda=\left(3^{3} \cdot 4^{2}\right)$ lattice. We find

$$
\begin{gathered}
\alpha_{a p}\left(\left(3^{3} \cdot 4^{2}\right)\right)=3.939 \pm 0.017 \\
\alpha_{0, a p}\left(\left(3^{3} \cdot 4^{2}\right)\right)=3.221 \pm 0.078
\end{gathered}
$$

and

$$
\beta_{a p}\left(\left(3^{3} \cdot 4^{2}\right)\right)=5.313 \pm 0.050
$$

\section{D. $\left(3^{2} \cdot 4 \cdot 3 \cdot 4\right)$ Lattice}

We present our results for the $\left(3^{2} \cdot 4 \cdot 3 \cdot 4\right)$ lattice in Tables XIX] XXIV. We have

$$
\begin{aligned}
& 3.922582062<\alpha\left(\left(3^{2} \cdot 4 \cdot 3 \cdot 4\right)\right)<3.9563647405 \\
& 3.142411228<\alpha_{0}\left(\left(3^{2} \cdot 4 \cdot 3 \cdot 4\right)\right)<3.299213098
\end{aligned}
$$

and

$$
5.264056522<\beta\left(\left(3^{2} \cdot 4 \cdot 3 \cdot 4\right)\right)<5.360035653
$$

We compute

$$
\begin{gathered}
\alpha_{a p}\left(\left(3^{2} \cdot 4 \cdot 3 \cdot 4\right)\right)=3.939 \pm 0.017 \\
\alpha_{0, a p}\left(\left(3^{2} \cdot 4 \cdot 3 \cdot 4\right)\right)=3.221 \pm 0.078
\end{gathered}
$$

and

$$
\beta_{a p}\left(\left(3^{2} \cdot 4 \cdot 3 \cdot 4\right)\right)=5.312 \pm 0.048
$$


Evidently, the upper and lower bounds on $\xi\left(\left(3^{3} \cdot 4^{2}\right)\right)$ are very close or equal (to the indicated number of significant figures) to the corresponding $\xi\left(\left(3^{2} \cdot 4 \cdot 3 \cdot 4\right)\right)$, where here $\xi(\Lambda)$ denotes $\alpha(\Lambda), \alpha_{0}(\Lambda)$, or $\beta(\Lambda)$, and so are the resultant approximate values. This presumably reflects the fact that a circuit around any vertex on these lattices contains the same number of triangles (namely three) and squares (namely two), and the only difference is the order in which these appear in the traversal.

\section{E. Summary of Bounds from Strip Calculations}

In Table XXV we list the approximate values of $\alpha_{a p}(\Lambda), \alpha_{0, a p}(\Lambda)$, and $\beta_{a p}(\Lambda)$ for these heteropolygonal Archimedean lattices, together with the corresponding quantities for homopolygonal Archimedean lattices. For the cases where we presented exact results in Ref. [1], namely $\alpha(\operatorname{tr} i), \alpha_{0}(\operatorname{tr} i)$, and $\beta(h c)$, we list these instead of the approximate values. As we found in [1] for homopolygonal Archimedean lattices, so also here we find, for these heteropolygonal Archimedean lattices, that our bounds and the resultant values of $\alpha_{a p}(\Lambda)$, $\alpha_{0, a p}(\Lambda)$, and $\beta_{a p}(\Lambda)$ are monotonically increasing functions of vertex degree (i.e., lattice coordination number), $\Delta(\Lambda)$.

Applying similar techniques, we have also obtained bounds on exponential growth constants for spanning forests and connected spanning subgraphs on heteropolygonal Archimedean lattices. Recall that a spanning forest in a graph $G$ is a spanning subgraph of $G$ that does not contain any circuits. Let us denote the number of spanning forests of a graph $G$ as $N_{S F}(G)$ and define $\phi(\{G\}) \equiv \lim _{n(G) \rightarrow \infty}\left[N_{S F}(G)\right]^{1 / n(G)}$. Recall that $N_{S F}(G)=T(G, 2,1)$. As an illustration, for the $\left(4 \cdot 8^{2}\right)$ lattice, we infer the bounds $2.779135 \leq \phi\left(\left(4 \cdot 8^{2}\right)\right) \leq 2.779486$. Since the upper and lower bounds are very close to each other, we determine the approximate value to be $\phi\left(\left(4 \cdot 8^{2}\right)\right)=2.77931 \pm 0.00018$. We will present these results elsewhere.

\section{CONNECTION WITH $W(\Lambda, q)$ BOUNDS FOR ARCHIMEDEAN LATTICES $\Lambda$}

The property that we find in our calculations, that $\alpha\left(\Lambda, L_{y}\right.$, free $)$ and $\alpha_{0}\left(\Lambda, L_{y}\right.$, free $)$ are monotonically increasing functions of strip width for a strip of the lattice $\Lambda$, is the reverse of the dependence on strip width that was found with $W\left(\Lambda, L_{y}\right.$, free, $\left.q\right)$ when the latter function is evaluated at $q \geq \chi(\Lambda)$, i.e., the range of $q$ required for a proper $q$-coloring of the lattice $\Lambda$ [23]. We interpret this as a consequence of the fact that $\alpha\left(\Lambda, L_{y}, f r e e\right)$ and $\alpha_{0}\left(\Lambda, L_{y}\right.$, free $)$ involve the evaluation of $|W(\Lambda, q)|$ at different values of $q$, namely $q=-1$ and $q=0$, respectively. 
In [1] we noted our observation concerning analytic expressions that were proved to be lower bounds on $W(\Lambda, q)$ for Archimedean lattices in [18] (see also [24]-[27]) with $q \geq \chi(\Lambda)$, using a coloring-matrix method that had been applied to derive a lower bound on $W(s q, q)$ in [28]. The observation was that, for a given Archimedean lattice $\Lambda$, if one sets $q=-1$ or $q=0$ in the analytic expressions that had been proved in [18] to be lower bounds on $W(\Lambda, q)$ for $q \geq \chi(\Lambda)$, then the resultant values are consistent with being upper bounds on $\alpha(\Lambda)$ and $\alpha_{0}(\Lambda)$, respectively. Therefore, we conjectured in [1] that these evaluations are, indeed, upper bounds on $\alpha(\Lambda)$ and $\alpha_{0}(\Lambda)$. We recall that the lower bound on $W(\Lambda, q)$ that was proved in [18], where $\Lambda$ is an Archimedean lattice, is

$$
W(\Lambda, q) \geq W(\Lambda, q)_{\ell}
$$

where (see Eq. (4.11) of [18])

$$
W\left(\left(\prod_{i} p_{i}^{a_{i}}\right), q\right)_{\ell}=\frac{\prod_{i}\left[D_{p_{i}}(q)\right]^{\nu_{p_{i}}}}{q-1},
$$

where $\nu_{p_{i}}$ was defined below Eq. (1.1),

$$
D_{n}(q)=\sum_{s=0}^{n-2}(-1)^{s}\left(\begin{array}{c}
n-1 \\
s
\end{array}\right) q^{n-2-s}
$$

and $q \geq \chi(\Lambda)$. The conjectured upper bounds on $\alpha(\Lambda)$ and $\alpha_{0}(\Lambda)$ are then as follows, where $\Lambda$ is an Archimedean lattice:

$$
\alpha(\Lambda)<\alpha_{u, w}(\Lambda)
$$

and

$$
\alpha_{0}(\Lambda)<\alpha_{0, u, w}(\Lambda)
$$

where

$$
\alpha_{u, w}(\Lambda)=\frac{\prod_{i}\left|D_{p_{i}}(-1)\right|^{\nu_{p_{i}}}}{2}
$$

and

$$
\alpha_{0, u, w}(\Lambda)=\prod_{i}\left|D_{p_{i}}(0)\right|^{\nu_{p_{i}}} .
$$

We list these below for each of the eleven Archimedean lattices, in order of increasing vertex degree, $\Delta(\Lambda)$, and for a given $\Delta(\Lambda)$, in order of increasing girth, $g(\Lambda)$. To indicate their connection with bounds on the $W$ function, we append a subscript $w$ :

$$
\alpha_{u, w}\left(\left(3 \cdot 12^{2}\right)\right)=\frac{3^{1 / 3} \times(2047)^{1 / 6}}{2}=2.569587414
$$




$$
\begin{gathered}
\alpha_{u, w}\left(\left(4 \cdot 8^{2}\right)\right)=\frac{7^{1 / 4} \times(127)^{1 / 4}}{2}=2.730206175 \\
\alpha_{u, w}((4 \cdot 6 \cdot 12))=\frac{7^{1 / 4} \times(31)^{1 / 6} \times(2047)^{1 / 12}}{2}=2.721017178 \\
\alpha_{u, w}\left(\left(6^{3}\right)\right) \equiv \alpha(h c)=\frac{\sqrt{31}}{2}=2.783882181 \\
\alpha_{u, w}((3 \cdot 6 \cdot 3 \cdot 6)) \equiv \alpha_{u, w}(k a g)=\frac{3^{2 / 3} \times(31)^{1 / 3}}{2}=3.2671675385 \\
\alpha_{u, w}((3 \cdot 4 \cdot 6 \cdot 4))=\frac{3^{1 / 3} \times 7^{1 / 2} \times(31)^{1 / 6}}{2}=3.381580457 \\
\alpha_{u, w}\left(\left(4^{4}\right)\right) \equiv \alpha_{u, w}(s q)=\frac{7}{2} \\
\alpha_{u, w}\left(\left(3^{3} \cdot 4^{2}\right)\right)=\alpha_{u, w}\left(\left(3^{2} \cdot 4 \cdot 3 \cdot 4\right)\right)=\frac{3 \sqrt{7}}{2}=3.968626967 \\
\alpha_{u, w}\left(\left(3^{4} \cdot 6\right)\right)=\frac{3^{4 / 3} \times(31)^{1 / 6}}{2}=3.834351826 \\
\alpha_{u, w}\left(\left(3^{6}\right)\right) \equiv \alpha_{u, w}(\operatorname{tri})=\frac{9}{2}
\end{gathered}
$$

and

$$
\begin{gathered}
\alpha_{0, u, w}\left(\left(3 \cdot 12^{2}\right)\right)=2^{1 / 3} \times(11)^{1 / 6}=1.878922121 \\
\alpha_{0, u, w}\left(\left(4 \cdot 8^{2}\right)\right)=3^{1 / 4} \times 7^{1 / 4}=2.140695143 \\
\alpha_{0, u, w}((4 \cdot 6 \cdot 12))=3^{1 / 4} \times 5^{1 / 6} \times(11)^{1 / 12}=2.101638609 \\
\alpha_{0, u, w}\left(\left(6^{3}\right)\right) \equiv \alpha_{0, u, w}(h c)=\sqrt{5}=2.2360679775 \\
\alpha_{0, u, w}((3 \cdot 6 \cdot 3 \cdot 6)) \equiv \alpha_{0, u, w}(k a g)=2^{2 / 3} \times 5^{1 / 3}=2.714417617 \\
\alpha_{0, u, w}((3 \cdot 4 \cdot 6 \cdot 4))=2^{1 / 3} \times 3^{1 / 2} \times 5^{1 / 6}=2.853638528 \\
\alpha_{0, u, w}\left(\left(4^{4}\right)\right) \equiv \alpha_{0, u, w}(s q)=3 \\
\alpha_{0, u, w}\left(\left(3^{3} \cdot 4^{2}\right)\right)=\alpha_{0, u, w}\left(\left(3^{2} \cdot 4 \cdot 3 \cdot 4\right)=2 \sqrt{3}=3.464101615\right. \\
\alpha_{0, u, w}\left(\left(3^{4} \cdot 6\right)\right)=2^{4 / 3} \times 5^{1 / 6}=3.295097945
\end{gathered}
$$

and

$$
\alpha_{0, u, w}\left(\left(3^{6}\right)\right) \equiv \alpha_{u, w, 0}(t r i)=4 .
$$

Using Eq. (4.18) of Ref. [18], we can slightly improve the suggested upper bounds for the $(4 \cdot 6 \cdot 12)$ lattice as follows:

$$
\alpha((4 \cdot 6 \cdot 12))_{u, w}=\frac{(42170569)^{1 / 12}}{2^{2 / 3}}=2.721014094
$$


and

$$
\alpha_{0}((4 \cdot 6 \cdot 12))_{u, w}=3^{1 / 6} \times(805)^{1 / 12}=2.097344955 .
$$

These numerical values are listed in Table XXVI. In this table we also list the exact values of $\tau(\Lambda)$ from [22], [29]-[31] (to the indicated number of significant figures).

\section{CONNECTION WITH $W\left(\Lambda_{d u a l}, q\right)$ BOUNDS ON DUALS OF ARCHIMEDEAN LATTICES}

\section{A. General}

To each Archimedean lattice $\Lambda=\left(\prod_{i} p_{i}^{a_{i}}\right)$, there corresponds a planar dual lattice $\Lambda_{\text {dual }}$ obtained by mapping the vertices and faces of $\Lambda$ to the faces and vertices, respectively, of $\Lambda_{\text {dual }}$. Just as all of the vertices of an Archimedean lattice are equivalent, all of the faces of the dual of an Archimedean lattice, i.e., the polygons of which it is comprised, are equivalent. The dual Archimedean lattice $\Lambda_{\text {dual }}$ is defined by the ordered product of degrees of vertices that one traverses in a circuit around the boundary of any face,

$$
\Lambda_{\text {dual }}=\left[\prod_{i} \Delta\left(v_{i}\right)^{b_{i}}\right]
$$

where in the above product, the notation $\Delta\left(v_{i}\right)^{b_{i}}$ indicates that the vertex $v_{i}$ with degree $\Delta\left(v_{i}\right)$ occurs contiguously $b_{i}$ times (and can also occur noncontiguously) in the circuit. We define $b_{i, s}=\sum_{i} b_{i}$. The polygons of which $\Lambda_{\text {dual }}$ is comprised have $p=b_{i, s}$ sides. For notational clarity, square brackets are used in Eq. (6.1) for dual Archimedean lattices, while parentheses are used in Eq. (1.1) for Archimedean lattices, so, e.g., the Archimedean lattice $\left(3 \cdot 12^{2}\right)$ has, as its (planar) dual, the $\left[3 \cdot 12^{2}\right]$ lattice, and so forth for others. The dual of an Archimedean lattice is also Archimedean if and only if the original lattice is homopolygonal. Specifically, the duality transformation maps the square lattice to an isomorphic copy of itself, and interchanges the triangular and honeycomb lattices, i.e., $\left[4^{4}\right]=\left(4^{4}\right)=(s q)$, $\left[3^{6}\right]=\left(6^{3}\right)=(h c)$, and $\left[6^{3}\right]=\left(3^{6}\right)=($ tri $)$. The duals of heteropolygonal Archimedean

lattices are not, themselves, Archimedean, since they are heterovertitial, i.e., contain vertices of different degrees. One can still define an effective vertex degree for these heterovertitial Archimedean dual lattices. Using $\Delta_{e f f}(\{G\})=\lim _{n(G) \rightarrow \infty} 2 e(G) / n(G)$ as discussed in the introduction, one has [18]

$$
\Delta_{e f f}\left(\Lambda_{d u a l}\right)=\nu_{p} p=\frac{2 p}{p-2}
$$


where

$$
\nu_{p}=\left[\sum_{i} \frac{b_{i, s}}{\Delta\left(v_{i}\right)}\right]^{-1}=\frac{2}{p-2} .
$$

Note that for a lattice that is $\Delta$-regular, the effective vertex degree just reduces to the uniform vertex degree. In particular, for the duals of homopolygonal $(h p)$ Archimedean lattices (which are thus $\Delta$-regular), $\Delta_{\text {eff }}\left(\Lambda_{\text {dual }, h p}\right)=\Delta\left(\Lambda_{\text {dual }, h p}\right)$.

\section{B. Values of $\beta_{u, w}\left(\Lambda_{\text {dual }}\right)$}

Using our inferred upper bounds on $\alpha(\Lambda)$ in conjunction with the duality relation (A15) and the values of $\nu(\Lambda)$ given below in Eqs. (A9)-(A11), we thus obtain inferred upper bounds on $\beta\left(\Lambda_{\text {dual }}\right)$ for Archimedean dual lattices. Since the conjectured upper bounds on $\alpha(\Lambda)$ and $\alpha_{0}(\Lambda)$ in Eqs. (5.4)-(5.7) are analytic, it is convenient to use them for this purpose. The resultant suggested upper bounds are of the form

$$
\beta\left(\Lambda_{\text {dual }}\right)<\beta_{u, w}\left(\Lambda_{\text {dual }}\right)
$$

where, as above, the subscript $w$ refers to the connection with $W(\Lambda, q)$. In order of increasing $p$ for duals of Archimedean lattices comprised of $p$-gons, we have

$$
\begin{gathered}
\beta_{u, w}\left(\left[3 \cdot 12^{2}\right]\right)=\left[\alpha_{u, w}\left(\left(3 \cdot 12^{2}\right)\right)\right]^{2}=\frac{3^{2 / 3} \times(2047)^{1 / 3}}{4}=6.602779477 \\
\beta_{u, w}\left(\left[4 \cdot 8^{2}\right]\right)=\left[\alpha_{u, w}\left(\left(4 \cdot 8^{2}\right)\right)\right]^{2}=\frac{7^{1 / 2} \times(127)^{1 / 2}}{4}=7.45402576 \\
\beta_{u, w}([4 \cdot 6 \cdot 12])=\left[\alpha_{u, w}((4 \cdot 6 \cdot 12))\right]^{2}=\frac{7^{1 / 2} \times(31)^{1 / 3} \times(2047)^{1 / 6}}{4}=7.403934483 \\
\beta_{u, w}\left(\left[6^{3}\right]\right)=\beta_{u, w}\left(\left(3^{6}\right)\right) \equiv \beta_{u, w}(\operatorname{tri})=\left[\alpha_{u, w}\left(\left(6^{3}\right)\right)\right]^{2}=\frac{31}{4}=7.75 \\
\beta_{u, w}([3 \cdot 6 \cdot 3 \cdot 6])=\alpha_{u, w}((3 \cdot 6 \cdot 3 \cdot 6))=\frac{3^{2 / 3} \times(31)^{1 / 3}}{2}=3.2671675385 \\
\beta_{u, w}([3 \cdot 4 \cdot 6 \cdot 4])=\alpha_{u, w}((3 \cdot 4 \cdot 6 \cdot 4))=\frac{3^{1 / 3} \times 7^{1 / 2} \times(31)^{1 / 6}}{2}=3.381580457 \\
\beta_{u, w}\left(\left[4^{4}\right]\right)=\alpha_{u, w}\left(\left(4^{4}\right)\right) \equiv \alpha_{u, w}(s q)=\frac{7}{2} \\
\beta_{u, w}\left(\left[3^{3} \cdot 4^{2}\right]\right)=\left[\alpha_{u, w}\left(\left(3^{3} \cdot 4^{2}\right)\right)\right]^{2 / 3}=\frac{3^{2 / 3} \times 7^{1 / 3}}{2^{2 / 3}}=2.50664897 \\
\beta_{u, w}\left(\left[3^{2} \cdot 4 \cdot 3 \cdot 4\right]\right)=\left[\alpha_{u, w}\left(\left(3^{2} \cdot 4 \cdot 3 \cdot 4\right)\right)\right]^{2 / 3}=\frac{3^{2 / 3} \times 7^{1 / 3}}{2^{2 / 3}}=2.50664897
\end{gathered}
$$




$$
\beta_{u, w}\left(\left[3^{4} \cdot 6\right]\right)=\left[\alpha_{u, w}\left(\left(3^{4} \cdot 6\right)\right)\right]^{2 / 3}=\frac{3^{8 / 9} \times(31)^{1 / 9}}{2^{2 / 3}}=2.44978501
$$

and

$$
\beta_{u, w}\left(\left[3^{6}\right]\right)=\beta_{u, w}\left(\left(6^{3}\right)\right) \equiv \beta_{u, w}(h c)=\left[\alpha_{u, w}\left(\left(3^{6}\right)\right)\right]^{1 / 2}=\frac{3}{\sqrt{2}}=2.12132034 .
$$

These values are listed in Table XXVII.

\section{Connection with $W\left(\Lambda_{\text {dual }}, q\right)$}

In addition to the lower bound for $W(\Lambda, q)$ on Archimedean lattices $\Lambda$, (5.1) with (5.2), Ref. [18] also proved a general lower bound for $W\left(\Lambda_{\text {dual }}, q\right)$ on dual Archimedean lattices $\Lambda_{\text {dual }}$. This lower bound, applicable for $q \geq \chi\left(\Lambda_{\text {dual }}\right)$, is

$$
W\left(\Lambda_{\text {dual }}, q\right) \geq W\left(\Lambda_{\text {dual }}, q\right)_{\ell}
$$

where (see Eq. (5.1) of [18])

$$
W\left(\left[\prod_{i} \Delta_{i}^{b_{i}}\right], q\right)_{\ell}=\frac{\left[D_{p}(q)\right]^{\nu_{p}}}{q-1} .
$$

As with Archimedean lattices, this naturally leads to the conjecture that evaluating Eq. (6.17) at $q=-1$ and $q=0$ would yield upper bounds on $\alpha\left(\Lambda_{\text {dual }}\right)$ and $\alpha_{0}\left(\Lambda_{\text {dual }}\right)$, respectively, i.e.,

$$
\alpha\left(\Lambda_{\text {dual }}\right)<\alpha_{u, w}\left(\Lambda_{\text {dual }}\right)
$$

and

$$
\alpha_{0}\left(\Lambda_{\text {dual }}\right)<\alpha_{0, u, w}\left(\Lambda_{\text {dual }}\right)
$$

where

$$
\alpha_{u, w}\left(\Lambda_{\text {dual }}\right)=\frac{\left|D_{p}(-1)\right|^{\nu_{p}}}{2}
$$

and

$$
\alpha_{0, u, w}\left(\Lambda_{\text {dual }}\right)=\left|D_{p}(0)\right|^{\nu_{p}}
$$

The values of $\alpha_{u, w}\left(\Lambda_{\text {dual }}\right)$ and $\alpha_{0, u, w}\left(\Lambda_{\text {dual }}\right)$ are listed below:

$$
\alpha_{u, w}\left(\Lambda_{\text {dual }}\right)=\frac{9}{2} \quad \text { and } \quad \alpha_{0, u, w}\left(\Lambda_{\text {dual }}\right)=4 \quad \text { for } \Lambda_{\text {dual }}=\left[3 \cdot 12^{2}\right], \quad\left[4 \cdot 8^{2}\right], \quad[4 \cdot 6 \cdot 12], \quad\left[6^{3}\right]
$$




$$
\alpha_{u, w}\left(\Lambda_{d u a l}\right)=\frac{7}{2} \quad \text { and } \quad \alpha_{0, u, w}\left(\Lambda_{d u a l}\right)=3 \quad \text { for } \Lambda_{d u a l}=[3 \cdot 6 \cdot 3 \cdot 6], \quad[3 \cdot 4 \cdot 6 \cdot 4], \quad\left[4^{4}\right]
$$

and

$$
\begin{aligned}
& \alpha_{u, w}\left(\Lambda_{\text {dual }}\right)=2^{-1} \times(15)^{2 / 3}=3.04110100 \text { and } \alpha_{0, u, w}\left(\Lambda_{\text {dual }}\right)=2^{4 / 3}=2.5198421 \\
& \text { for } \Lambda_{\text {dual }}=\left[3^{3} \cdot 4^{2}\right], \quad\left[3^{2} \cdot 4 \cdot 3 \cdot 4\right], \quad\left[3^{4} \cdot 6\right]
\end{aligned}
$$

As implied by duality, for the homopolygonal Archimedean lattices the values of $\alpha_{u, w}\left(\left[3^{6}\right]\right)=$ $\alpha_{u, w}\left(\left(6^{3}\right)\right)=\alpha_{u, w}(h c)$ and $\alpha_{0, u, w}\left(\left[3^{6}\right]\right)=\alpha_{0, u, w}\left(\left(6^{3}\right)\right)=\alpha_{0, u, w}(h c)$ are the same as those given above in Eqs. (5.11) and (5.21); the values of $\alpha_{u, w}\left(\left[4^{4}\right]\right)=\alpha_{u, w}(s q)$ and $\alpha_{0, u, w}\left(\left[4^{4}\right]\right)=$ $\alpha_{0, u, w}(s q)$ are the same as those given in Eqs. (5.14) and (5.24); and the values of $\alpha_{u, w}\left(\left[6^{3}\right]\right)=$ $\alpha_{u, w}\left(\left(3^{6}\right)\right)=\alpha_{u, w}($ tri $)$ and $\alpha_{0, u, w}\left(\left[6^{3}\right]\right)=\alpha_{0, u, w}\left(\left(3^{6}\right)\right)_{u w}=\alpha_{0, u, w}($ tri $)$ are the same as those given in Eqs. (5.17) and (5.27).

Using different paths for the operation of the coloring matrix, Ref. [18] also derived more stringent lower bounds on $W\left(\Lambda_{\text {dual }}\right)$ for certain dual heteropolygonal Archimedean lattices, again applicable for $q \geq \chi\left(\Lambda_{\text {dual }}\right)$ :

$$
\begin{gathered}
W\left(\left[3 \cdot 12^{2}\right], q\right) \geq \frac{[(q-2)(q-3)]^{2 / 3}}{(q-1)^{1 / 3}} \\
W\left(\left[4 \cdot 8^{2}\right], q\right) \geq\left[\frac{(q-2)\left(q^{2}-5 q+7\right)}{q-1}\right]^{1 / 2}
\end{gathered}
$$

and

$$
W([4 \cdot 6 \cdot 12], q) \geq \frac{(q-2)\left(q^{2}-5 q+7\right)^{1 / 3}}{(q-1)^{2 / 3}} .
$$

Following the same procedure as explained above, we can use these to obtain more restrictive conjectured upper bounds on $\xi\left(\Lambda_{\text {dual }}\right)$ for $\xi=\alpha, \alpha_{0}$ and $\Lambda_{\text {dual }}=\left[3 \cdot 12^{2}\right],\left[4 \cdot 8^{2}\right]$, and $[4 \cdot 6 \cdot 12]$. These are marked with primes to distinguish them from the upper bounds given above:

$$
\begin{gathered}
\alpha_{u, w^{\prime}}\left(\left[3 \cdot 12^{2}\right]\right)=2 \times 3^{2 / 3}=4.160168 \\
\alpha_{0, u, w^{\prime}}\left(\left[3 \cdot 12^{2}\right]\right)=6^{2 / 3}=3.301927 \\
\alpha_{u, w^{\prime}}\left(\left[4 \cdot 8^{2}\right]\right)=\sqrt{\frac{39}{2}}=4.415880 \\
\alpha_{0, u, w^{\prime}}\left(\left[4 \cdot 8^{2}\right]\right)=\sqrt{14}=3.741657
\end{gathered}
$$




$$
\alpha_{u, w^{\prime}}([4 \cdot 6 \cdot 12])=2^{-2 / 3} \times 3 \times 13^{1 / 3}=4.443744
$$

and

$$
\alpha_{0, u, w^{\prime}}([4 \cdot 6 \cdot 12])=2 \times 7^{1 / 3}=3.825862 .
$$

The values of $\alpha_{u, w}\left(\Lambda_{d u a l}\right)$ and $\alpha_{0, u, w}\left(\Lambda_{d u a l}\right)$, or the more restrictive values $\alpha_{u, w^{\prime}}\left(\Lambda_{d u a l}\right)$ and $\alpha_{0, u, w^{\prime}}\left(\Lambda_{\text {dual }}\right)$ where they apply, are listed in Table XXVII.

We recall that the lower bounds that were proved to apply for $W(\Lambda, q)$ for $q \geq \chi(\Lambda)$ on all Archimedean lattices $\Lambda$ in [18] were found to be very close to the actual values of $W(\Lambda, q)$ as determined by Monte-Carlo simulations and series expansions (and by the exact result for $W($ tri $)$ in [32]). This led to the expectation that, not only would (5.6) and (5.7) constitute upper bounds on $\alpha(\Lambda)$ and $\alpha_{0}(\Lambda)$, respectively (which our results support), but also that the values of $\alpha_{u, w}(\Lambda)$ and $\alpha_{0, u, w}(\Lambda)$ would be close to the actual values of $\alpha(\Lambda)$ and $\alpha_{0}(\Lambda)$. This expectation was confirmed for homopolygonal Archimedean lattices in [1]. Here, we also confirm this for the heteropolygonal Archimedean lattices for which we have obtained approximate values of these exponential growth constants, namely for the $\left(4 \cdot 8^{2}\right),(3 \cdot 6 \cdot 3 \cdot 6)$, $\left(3^{3} \times 4^{2}\right)$, and $\left(3^{2} \cdot 4 \cdot 3 \cdot 4\right)$ lattices.

Furthermore, by duality, one can calculate values of $\beta_{u, w}(\Lambda)$ on Archimedean lattices corresponding to these values of $\alpha_{u, w}\left(\Lambda_{\text {dual }}\right)$ on duals of Archimedean lattices. We obtain

$$
\begin{aligned}
& \beta_{u, w}(\Lambda)=\left[\alpha_{u, w}\left(\Lambda_{\text {dual }}\right)\right]^{1 / 2}=\frac{3}{\sqrt{2}}=2.121320 \\
& \text { for } \Lambda=\left(3 \cdot 12^{2}\right), \quad\left(4 \cdot 8^{2}\right), \quad(4 \cdot 6 \cdot 12), \quad\left(6^{3}\right) \\
& \beta_{u, w}(\Lambda)=\alpha_{u, w}\left(\Lambda_{\text {dual }}\right)=\frac{7}{2} \\
& \text { for } \Lambda=(3 \cdot 6 \cdot 3 \cdot 6), \quad(3 \cdot 6 \cdot 4 \cdot 6), \quad\left(4^{4}\right) \\
& \beta_{u, w}(\Lambda)=\left[\alpha_{u, w}\left(\Lambda_{\text {dual }}\right)\right]^{3 / 2}=\frac{15}{2 \sqrt{2}}=5.303301 \\
& \text { for } \Lambda=\left(3^{3} \cdot 4^{2}\right), \quad\left(3^{2} \cdot 4 \cdot 3 \cdot 4\right), \quad\left(3^{4} \cdot 6\right)
\end{aligned}
$$

and

$$
\beta_{u, w}\left(3^{6}\right) \equiv \beta_{u, w}(\operatorname{tri})=\left[\alpha_{u, w}(h c)\right]^{2}=\frac{31}{4}=7.75
$$

Using duality together with the values of $\alpha_{u, w^{\prime}}\left(\left[3 \cdot 12^{2}\right]\right), \alpha_{u, w^{\prime}}\left(\left[4 \cdot 8^{2}\right]\right)$, and $\alpha_{u, w^{\prime}}([4 \cdot 6 \cdot 12])$, we obtain the more restrictive conjectured upper limits

$$
\beta_{u, w^{\prime}}\left(\left(3 \cdot 12^{2}\right)\right)=\left[\alpha_{u, w^{\prime}}\left(\left[3 \cdot 12^{2}\right]\right)\right]^{1 / 2}=2^{1 / 2} \times 3^{1 / 3}=2.039649
$$




$$
\beta_{u, w^{\prime}}\left(\left(4 \cdot 8^{2}\right)\right)=\left[\alpha_{u, w^{\prime}}\left(\left[4 \cdot 8^{2}\right]\right)\right]^{1 / 2}=\left(\frac{39}{2}\right)^{1 / 4}=2.101400
$$

and

$$
\beta_{u, w^{\prime}}((4 \cdot 6 \cdot 12))=\left[\alpha_{u, w^{\prime}}([4 \cdot 6 \cdot 12])\right]^{1 / 2}=2^{-1 / 3} \times 3^{1 / 2} \times(13)^{1 / 16}=2.108019 .
$$

We list these values of $\beta_{u, w}(\Lambda)$ or $\beta_{u, w^{\prime}}(\Lambda)$ in Table XXVI. Since the application of the duality transformation twice is the identity map, it follows that for the homopolygonal Archimedean lattices, the values of $\beta_{u, w}(h c), \beta_{u, w}(s q)$, and $\beta_{u, w}($ tri $)$ in Eqs. (6.34), (6.35), and (6.37) are equal to the values obtained in Eqs. (6.15), (6.11), and (6.8).

We observe that where we can compare the values of $\beta_{u, w}(\Lambda)$ or $\beta_{u, w^{\prime}}(\Lambda)$ with the values $\beta_{a p}(\Lambda)$ that we have determined above via calculations with infinite-length, finite-width strips, namely for the $\left(4 \cdot 8^{2}\right),(3 \cdot 6 \cdot 3 \cdot 6),\left(3^{3} \times 4^{2}\right)$, and $\left(3^{2} \cdot 4 \cdot 3 \cdot 4 \cdot 3\right)$ lattices, they are reasonably close for each $\Lambda$. We will use this finding below. Interestingly, for the $\left(3^{3} \cdot 4^{2}\right)$ and $\left(3^{2} \cdot 4 \cdot 3 \cdot 4\right)$ lattices, the common value of $\beta_{u, w}\left(\left(3^{3} \cdot 4^{2}\right)\right)=\beta_{u, w}\left(\left(3^{2} \cdot 4 \cdot 3 \cdot 4\right)\right)=5.303301$ in Eq. (6.36) lies slightly below the upper bounds that we infer for these lattices from our computations with infinite-length, finite-width strips.

\section{COMPARATIVE ANALYSIS}

With these calculations on heteropolygonal Archimedean lattices, we extend our results in [1] for homopolygonal Archimedean lattices to the full set of Archimedean lattices. We find that for all Archimedean lattices, the values of $\alpha(\Lambda), \alpha_{0}(\Lambda)$, and $\beta(\Lambda)$ that are consistent with our inferred upper and lower bounds, and the exact values where we have calculated them, are monotonically increasing functions of $\Delta(\Lambda)$. In particular, this applies to the average values, $\alpha_{\text {ave }}(\Lambda), \alpha_{0, a v e}(\Lambda)$, and $\beta_{\text {ave }}(\Lambda)$ and to the $\alpha_{u, w}(\Lambda), \alpha_{0, u, w}(\Lambda)$, and $\beta_{u, w}(\Lambda)$ values. These statements are also true of our results for the dual Archimedean lattices $\Lambda_{\text {dual }}$. We recall that for a $\Delta$-regular graph $G$, the number of edges is related to the number of vertices by $e(G)=(\Delta / 2) n$, so the exponential growth constant $\epsilon(\{G\})$ increases with $\Delta$, as $\epsilon(\{G\})=2^{\Delta / 2}$. Thus, the monotonic increase that we find for $\alpha(\Lambda), \alpha_{0}(\Lambda)$, and $\beta(\Lambda)$ as functions of $\Delta(\Lambda)$ on these lattices can be interpreted as a consequence of the fact that, for a section $G_{\Lambda}$ of the lattice $\Lambda$ with $n\left(G_{\Lambda}\right) \rightarrow \infty$, an increase in $\Delta\left(G_{\Lambda}\right)$ leads, via the exponential increase in $N_{e o}\left(G_{\Lambda}\right)$, to a commensurately large exponential increase in $a\left(G_{\Lambda}\right), a_{0}\left(G_{\Lambda}\right)$, and $b\left(G_{\Lambda}\right)$.

In [1] we observed that the increase in these exponential growth constants with vertex degree $\Delta(\Lambda)$ for the homopolygonal Archimedean lattices is the opposite of what was found for the the behavior of $W(\Lambda, q)$ for these lattices $\Lambda$ with $q$ in the range $q \geq \chi(\Lambda)$ used for 
proper q-coloring. In [24] (see, e.g., Fig. 5), $W(\Lambda, q)$ was shown to be a monotonically decreasing function of $\Delta(\Lambda)$ for $q \geq \chi(\Lambda)$. This dependence was also shown for the upper and lower bounds on $W(\Lambda, q)$ for Archimedean lattices, including heteropolygonal lattices, in [18, 25, 26] (see also [24, 27]). This is a consequence of the property that an increase in $\Delta(\Lambda)$ generically increases the constraints on a proper $q$-coloring of the lattice $\Lambda$ [18, 23, 24 ]. The reversal in the dependence of $W(\Lambda, q)$ on $\Delta(\Lambda)$ when one switches from $q \geq \chi(\Lambda)$ to $q \leq 0$ was seen in (Fig. 5 of) Ref. [24]. Here we have extended this contrast from the homopolygonal lattices studied in [1] to heteropolygonal Archimedean lattices, and hence to all Archimedean lattices.

As we did for homopolygonal Archimedean lattices in [1], we next compare our results for $\alpha(\Lambda), \alpha_{0}(\Lambda)$, and $\beta(\Lambda)$ with the exponential growth constants for spanning trees on heteropolygonal Archimedean lattices. We first review the relevant definitions. A tree graph is a connected graph that does not contain any circuits, and a spanning tree of a graph $G$ is a subgraph of $G$ that is a tree and that contains all of the vertices of $G$. From Eq. (A23), it follows that the number of spanning trees in a graph $G$, denoted $N_{S T}(G)$, is given by the following evaluation of the Tutte polynomial:

$$
N_{S T}(G)=T(G, 1,1) .
$$

A different way to calculate $N_{S T}(G)$, which has been the basis of a number of exact calculations, starts with the adjacency matrix $A$ of the graph $G$. Let us denote the number of edges connecting two adjacent vertices $v_{i}$ and $v_{j}$ as $N\left(e_{i j}\right)$. The adjacency matrix $A$ is an $n(G) \times n(G)$ matrix with elements $A_{i j}=N\left(e_{i j}\right)$ if the vertices $v_{i}$ and $v_{j}$ are adjacent and $A_{i j}=0$ otherwise. The Laplacian matrix $Q$ is an $n(G) \times n(G)$ matrix with elements $Q_{i j}=\Delta(G) \delta_{i j}-A_{i j}$, where $\delta_{i j}$ is the Kronecker delta function. The sum of the elements in any row or column of $Q$ is zero, and consequently, one of the eigenvalues of $Q$ is zero. Denote the remaining eigenvalues as $\lambda_{1}^{(Q)}, \ldots, \lambda_{n(G)-1}^{(Q)}$. Then [2]

$$
N_{S T}(G)=\frac{1}{n(G)} \prod_{s=1}^{n(G)-1} \lambda_{s}^{(Q)}
$$

For the lattice graphs $G$ studied here, $N_{S T}(G)$ grows exponentially rapidly with the number of vertices, $n(G)$. It is then natural to define the corresponding exponential growth constant,

$$
\tau(\{G\})=\lim _{n(G) \rightarrow \infty}\left[N_{S T}(G)\right]^{1 / n(G)} .
$$

An equivalent quantity that has often been used in previous works is $z(\{G\})=\ln [\tau(\{G\})]$. For a lattice graph, in the limit $n(G) \rightarrow \infty$, the logarithm of the product of eigenvalues in Eq. 
(7.2), which gives $z(\Lambda)$, becomes an integral, whose integrand is determined from a knowledge of the basis vectors of the lattice (see Eq. (4.16) in [30]). This integral formulation has been used for the exact calculation of $z(\Lambda)$, or equivalently, $\tau(\Lambda)$ for all of the Archimedean lattices. Specifically, $z(\Lambda)$ was calculated for the square, triangular, and honeycomb lattices in [29]; for the $\left(3 \cdot 12^{2}\right)$ and $(3 \cdot 6 \cdot 3 \cdot 6)$ lattices in [30]; for the $\left(4 \cdot 8^{2}\right)$ lattice in [30, 31]; and for the $(4 \cdot 6 \cdot 12),(3 \cdot 4 \cdot 6 \cdot 4),\left(3^{3} \cdot 4^{2}\right),\left(3^{2} \cdot 4 \cdot 3 \cdot 4\right)$, and $\left(3^{4} \cdot 6\right)$ lattice in [22]. We list the numerical values of $\tau(\Lambda)$ for these lattices in Table XXVI. By duality, the values of $\tau\left(\Lambda_{\text {dual }}\right)$ on the dual Archimedean lattices are exactly determined in terms of these $\tau(\Lambda)$ values. We list the numerical values of $\tau\left(\Lambda_{\text {dual }}\right)$ in Table XXVII.

A theorem of Thomassen [33] states that if $G$ is a $\Delta$-regular graph of degree $\Delta(G) \leq 3$ which has no loops (but which may have bridges and multiple edges), then $N_{S T}(G) \leq a(G)$. Considering a family of graphs of this type and taking the limit $n(G) \rightarrow \infty$, this implies that in this limit, $\tau(\{G\}) \leq \alpha(\{G\})$. As must be true, in agreement with this theorem (for the special case relevant to our application to graphs without multiple edges), our result for the approximate value of $\alpha\left(\left(4 \cdot 8^{2}\right)\right)$ in Eq. (4.11) is greater than the exact result for $\tau\left(\left(4 \cdot 8^{2}\right)\right)$ from [30, 31], as listed in Table XXV. The values of $\alpha_{u, w}(\Lambda)$ for all of the $\Delta(\Lambda)=3$ Archimedean lattices are also in agreement with this theorem, as is evident from Table XXVI.

In [1] we observed that our determinations of $\alpha(\Lambda)$ and $\beta(\Lambda)$ on the homopolygonal Archimedean lattices $\Lambda=h c, s q$, tri were in agreement with an inequality on exponential growth constants implied by the Merino-Welsh conjecture [12]. Here we extend our investigation of this subject to the set of all Archimedean lattices. We first recall the Merino-Welsh conjecture. Let $G$ be a connected graph without loops or bridges (which may have multiple edges, although we restrict here to graphs without multiple edges). Then the Merino-Welsh conjecture (MWC) is the inequality [12]

$$
N_{S T}(G) \leq \max [a(G), b(G)], \quad \text { i.e., } T(G, 1,1) \leq \max [T(G, 2,0), T(G, 0,2)] \quad(\mathrm{MWC}) .
$$

In the later paper [34], Conde and Merino conjectured the stronger inequality that if $G$ is a connected graph without loops or bridges (which may have multiple edges), then

$$
\left[N_{S T}(G)\right]^{2} \leq a(G) b(G) \text {, i.e., }[T(G, 1,1)]^{2} \leq T(G, 2,0) T(G, 0,2) \quad(\mathrm{CMC}),
$$

where our abbreviation CMC stands for Conde-Merino conjecture. Some relevant related papers include [35]-38]. For our purposes, we first observe that the Merino-Welsh and Conde-Merino conjectures imply the following inequalities on exponential growth constants, where $\{G\}$ is the $n(G) \rightarrow \infty$ limit of graphs $G$ that satisfy the premise of the MWC and CMC:

$$
\tau(\{G\}) \leq \max [\alpha(\{G\}), \beta(\{G\})] \text { from MWC }
$$


and

$$
[\tau(\{G\})]^{2} \leq \alpha(\{G\}) \beta(\{G\}) \text { from CMC }
$$

For the comparison, we make use of the approximate values $\alpha_{a p}(\Lambda), \alpha_{0, a p}(\Lambda)$, and $\beta_{a p}(\Lambda)$ that we have determined from our upper and lower bounds for the $\left(4 \cdot 8^{2}\right),(3 \cdot 6 \cdot 3 \cdot 6)$, $\left(3^{3} \cdot 4^{2}\right)$, and $\left(3^{2} \cdot 4 \cdot 3 \cdot 4\right)$ Archimedean lattices. As is evident in Table XXV these are in agreement with the inequalities (7.6) and (7.7) implied, respectively, by the Merino-Welsh conjecture and the Conde-Merino conjecture. Our results in [1] and here are also useful as a quantitative measure of how close to being saturated the inequalities (7.6) and (7.7) are.

If we assume that for the other heteropolygonal Archimedean lattices, our values of $\alpha_{u, w}(\Lambda), \beta_{u, w}(\Lambda)$, and $\beta_{u, w^{\prime}}(\Lambda)$ are close to the actual respective values, then we can substitute these into (7.6) together with the known values of $\tau(\Lambda)$ for comparisons. As is evident in Table XXVT, in all cases, this comparison agrees with the inequalities (7.6) and (7.7) implied by the Merino-Welsh and Conde-Merino conjectures. This is also true of our results for the dual Archimedean lattices $\Lambda_{\text {dual }}$, as one can see from Table XXVII.

Moreover, with the same assumptions as above, we can combine our calculations on Archimedean lattices to comment on the relative sizes of $\alpha(\Lambda), \beta(\Lambda)$, and $\tau(\Lambda)$ as functions of $\Delta(\Lambda)$. We find

$$
\begin{gathered}
\alpha(\Lambda)>\tau(\Lambda)>\beta(\Lambda) \text { for } \Delta(\Lambda)=3 \\
\alpha(\Lambda), \beta(\Lambda)>\tau(\Lambda) \text { for } \Delta(\Lambda)=4
\end{gathered}
$$

and

$$
\beta(\Lambda)>\tau(\Lambda)>\alpha(\Lambda) \text { for } \Delta(\Lambda)=5,6
$$

Although the duals of heteropolygonal Archimedean lattice are not $\Delta$-regular, i.e., have vertices of different degrees, one can explore the dependence of these exponential growth constants on the effective vertex degree $\Delta_{\text {eff }}\left(\Lambda_{\text {dual }}\right)$ given in Eq. (6.2). With the same assumptions as above, we find similar inequalities for $\alpha_{u, w}\left(\Lambda_{d u a l}\right), \beta_{u, w}\left(\Lambda_{d u a l}\right)$ and the exactly known $\tau\left(\Lambda_{\text {dual }}\right)$ values (including also our results on homopolygonal lattices).

$$
\begin{gathered}
\alpha\left(\Lambda_{\text {dual }}\right)>\tau\left(\Lambda_{\text {dual }}\right)>\beta\left(\Lambda_{\text {dual }}\right) \quad \text { for } \Delta_{\text {eff }}\left(\Lambda_{\text {dual }}\right)=3, \frac{10}{3}, \\
\alpha\left(\Lambda_{\text {dual }}\right), \beta\left(\Lambda_{\text {dual }}\right)>\tau\left(\Lambda_{\text {dual }}\right) \quad \text { for } \Delta_{\text {eff }}\left(\Lambda_{\text {dual }}\right)=4
\end{gathered}
$$

and

$$
\beta\left(\Lambda_{\text {dual }}\right)>\tau\left(\Lambda_{\text {dual }}\right)>\alpha\left(\Lambda_{\text {dual }}\right) \text { for } \Delta_{\text {eff }}\left(\Lambda_{\text {dual }}\right)=6
$$




\section{CONCLUSIONS}

In this paper, extending our study in [1], we have inferred upper and lower bounds on the exponential growth constants $\alpha(\Lambda), \alpha_{0}(\Lambda)$, and $\beta(\Lambda)$ that characterize the asymptotic behavior of acyclic orientations, acyclic orientations with a single source vertex, and totally cyclic orientations of heteropolygonal Archimedean lattices. To our knowledge, these are the best bounds on these quantities. As in the case of the homopolygonal Archimedean lattices (honeycomb, square, and triangular), these bounds converge quickly, even for moderate values of $L_{y}$, the strip width. Furthermore, again as with the homopolygonal lattices, the upper and lower bounds are close to each other, which enables us to infer approximate values of the actual exponential growth constants themselves. A general property that we observe is that $\alpha(\Lambda), \alpha_{0}(\Lambda)$, and $\beta(\Lambda)$ are monotonically increasing functions of vertex degree $\Delta(\Lambda)$ for all Archimedean lattices, both homopolygonal and heteropolygonal. We have conjectured that analytic expressions that were proved in [18] to be lower bounds on $W(\Lambda, q)$ for values of $q$ used in proper $q$-colorings of Archimedean and dual Archimedean lattices $\Lambda$ and $\Lambda_{\text {dual }}$ provide upper bounds on $\alpha(\Lambda), \alpha_{0}(\Lambda), \alpha\left(\Lambda_{\text {dual }}\right)$, and $\alpha_{0}\left(\Lambda_{\text {dual }}\right)$. We have also used duality relations to obtain corresponding conjectured upper bounds on $\beta(\Lambda)$ and $\beta\left(\Lambda_{\text {dual }}\right)$. In all cases, these are consistent with the upper bounds that we derive from our calculations using infinite-length, finite-width lattice strips of these graphs. We have also made comparisons with the exponential growth constants for spanning trees on these lattices, finding agreement with inequalities that follow from the Merino-Welsh and CondeMerino conjectures. In addition to providing support for these inequalities, our results give a quantitative measure of how close to being saturated they are for the lattices that we study.

\section{Acknowledgments}

This research was supported in part by the Taiwan Ministry of Science and Technology grant MOST 103-2918-I-006-016 (S.-C.C.) and by the U.S. National Science Foundation grant No. NSF-PHY-16-1620628 (R.S.).

\section{Appendix A: Some Graph Theory Background}

In this appendix we briefly list some formulas that are relevant to our analysis in the text. As stated in the text, we denote a graph as $G=(V, E)$ with vertex and edge sets $V$ and $E$. We let $n=n(G)=|V|, e(G)=|E|, f c(G)$, and $k(G)$ denote the number of 
vertices, edges, faces, and connected components of $G$, respectively. The degree of a vertex in a graph is the number of edges that connect to it. Graphs whose vertices all have the same degree $\Delta$ is called a $\Delta$-regular graph. The girth $g(G)$ of a graph is the length of edges in a minimal-distance circuit on $G$. (If $G$ has no circuits, then $g(G)$ is not defined.)

An Archimedean lattice is a tiling of the (infinite) plane with one or more types of regular polygons (i.e., polygons whose sides all have equal length and whose internal angles are all equal) such that all vertices are equivalent. As discussed in the text, this means that an Archimedean lattice $\Lambda$ can be defined as the ordered sequence of polygons that one traverses in a circuit around any vertex, $\Lambda=\prod_{i} p_{i}^{a_{i}}$, where the $i$ 'th polygon has $p_{i}$ sides and appears $a_{i}$ times together in the sequence. There are eleven Archimedean lattices. Of these, three are homopolygonal, namely $\left(4^{4}\right)$ (square), $\left(3^{6}\right)$ (triangular), and $\left(6^{3}\right)$ (honeycomb), and the other eight are heteropolygonal. Synonymous notations include $\left(3 \cdot 12^{2}\right) \equiv(3 \cdot 12 \cdot 12)$, $\left(4 \cdot 8^{2}\right) \equiv(4 \cdot 8 \cdot 8)$, etc. The Archimedean lattices, listed in order of increasing vertex degree $\Delta(\Lambda)$, and, for a given $\Delta(\Lambda)$ in order of increasing girth $g(\Lambda)$, are

$$
\begin{aligned}
& \Delta(\Lambda)=3: \quad\left(3 \cdot 12^{2}\right), \quad(4 \cdot 8)^{2}, \quad(4 \cdot 6 \cdot 12), \quad(6)^{3} \\
& \Delta(\Lambda)=4: \quad(3 \cdot 6 \cdot 3 \cdot 6), \quad(3 \cdot 4 \cdot 6 \cdot 4), \quad\left(4^{4}\right) \\
& \Delta(\Lambda)=5: \quad\left(3^{3} \cdot 4^{2}\right), \quad\left(3^{2} \cdot 4 \cdot 3 \cdot 4\right), \quad\left(3^{4} \cdot 6\right),
\end{aligned}
$$

and

$$
\Delta(\Lambda)=6: \quad\left(3^{6}\right)
$$

Let $G$ be a planar graph, indicated as $G_{p l}$, and denote $G_{p l}^{*}$ as the (planar) dual graph. Then the Tutte polynomial satisfies $T\left(G_{p l}, x, y\right)=T\left(G_{p l}^{*}, y, x\right)$. Consequently, $a\left(G_{p l}\right)=b\left(G_{p l}^{*}\right)$. From duality, one has the equality $n\left(G_{p l}^{*}\right)=f c\left(G_{p l}\right)$. Recall the Euler relation that for a planar graph $G_{p l}, f c\left(G_{p l}\right)-e\left(G_{p l}\right)+n\left(G_{p l}\right)=2$. Following the notation in [30], for a $\Delta$-regular planar graph $G_{p l}$, we define the ratio

$$
\nu_{\left\{G_{p l}\right\}} \equiv \lim _{n(G) \rightarrow \infty} \frac{n\left(G_{p l}^{*}\right)}{n\left(G_{p l}\right)}=\lim _{n(G) \rightarrow \infty} \frac{f c\left(G_{p l}\right)}{n\left(G_{p l}\right)} .
$$

Using the Euler relation and the fact that $\Delta(G)=2 e(G) / n(G)$, we have

$$
\nu_{\left\{G_{p l}\right\}}=\frac{\Delta\left(G_{p l}\right)}{2}-1 \text {. }
$$

If the vertices of $G_{p l}^{*}$ have uniform degree, then $\nu\left(G_{p l}^{*}\right)=1 / \nu\left(G_{p l}\right)$. In general, even if the vertices of $G_{p l}^{*}$ do not have uniform degree, in the limit $n(G) \rightarrow \infty$,

$$
\nu\left(\left\{G_{p l}^{*}\right\}\right)=\frac{1}{\nu(\{G\})} .
$$


For homopolygonal lattices,

$$
\nu(s q)=1, \quad \nu(h c)=\frac{1}{\nu(\text { tri })}=\frac{1}{2} .
$$

For heteropolygonal lattices, we have

$$
\begin{gathered}
\nu\left(\left(3 \cdot 12^{2}\right)\right)=\nu\left(\left(4 \cdot 8^{2}\right)\right)=\nu((4 \cdot 6 \cdot 12))=\frac{1}{2} \\
\nu((3 \cdot 6 \cdot 3 \cdot 6))=\nu((3 \cdot 4 \cdot 6 \cdot 4)=1
\end{gathered}
$$

and

$$
\nu\left(\left(3^{3} \cdot 4^{2}\right)\right)=\nu\left(\left(3^{2} \cdot 4 \cdot 3 \cdot 4\right)\right)=\nu\left(\left(3^{4} \cdot 6\right)\right)=\frac{3}{2} .
$$

For a $\Delta$-regular planar graph $G_{p l}$, in the $n\left(G_{p l}\right) \rightarrow \infty$ limit,

$$
\begin{aligned}
\beta\left(\left\{G_{p l}\right\}\right) & =\lim _{n\left(G_{p l}\right) \rightarrow \infty}\left[T\left(G_{p l}, 0,2\right)\right]^{\frac{1}{n\left(G_{p l}\right)}}=\lim _{n\left(G_{p l}^{*}\right) \rightarrow \infty}\left[T\left(G_{p l}^{*}, 2,0\right)\right]^{\frac{\nu\left(G_{p l}\right)}{n\left(G_{p l}^{*}\right)}} \\
& =\left[\alpha\left(\left\{G_{p l}^{*}\right\}\right)\right]^{\nu\left(G_{p l}\right)} .
\end{aligned}
$$

Similarly,

$$
\left.\alpha\left(\left\{G_{p l}\right\}\right)\right)=\left[\beta\left(\left\{G_{p l}^{*}\right\}\right)\right]^{\nu\left(G_{p l}\right)} \text {, i.e., } \quad \beta\left(\left\{G_{p l}^{*}\right\}\right)=\left[\alpha\left(\left\{G_{p l}\right\}\right)\right]^{1 / \nu\left(G_{p l}\right)}
$$

In [1] we appliled these relations to determine $\beta(h c)$ in terms of $\alpha(\operatorname{tr} i)$, using

$$
\beta(h c)=[\alpha(\text { tri })]^{\nu(h c)}=[\alpha(\text { tri })]^{1 / 2}
$$

Here we will use these relations to determine $\beta\left(\Lambda_{\text {dual }}\right)$ for the duals of heteropolygonal lattices. In general, for each of the duals of Archimedean lattices, $\Lambda_{\text {dual }}$, we have

$$
\beta\left(\Lambda_{\text {dual }}\right)=[\alpha(\Lambda)]^{\frac{1}{\nu(\Lambda)}} .
$$

Specifically,

$$
\begin{gathered}
\beta\left(\Lambda_{\text {dual }}\right)=[\alpha(\Lambda)]^{2} \quad \text { for } \Lambda=\left(3 \cdot 12^{2}\right), \quad\left(4 \cdot 8^{2}\right), \quad(4 \cdot 6 \cdot 12) \\
\left.\beta\left(\Lambda_{\text {dual }}\right)\right)=\alpha(\Lambda) \quad \text { for } \Lambda=(3 \cdot 6 \cdot 3 \cdot 6), \quad(3 \cdot 4 \cdot 6 \cdot 4)
\end{gathered}
$$

and

$$
\beta\left(\Lambda_{\text {dual }}\right)=[\alpha(\Lambda)]^{2 / 3} \quad \text { for } \Lambda=\left(3^{3} \cdot 4^{2}\right), \quad\left(3^{2} \cdot 4 \cdot 3 \cdot 4\right), \quad\left(3^{4} \cdot 6\right) .
$$

Given a graph $G$, a spanning subgraph of $G$, denoted $G^{\prime}$, is a graph with the same vertex set $V$ and a subset of the edge set $E$, i.e., $G^{\prime}=G^{\prime}\left(V, E^{\prime}\right)$ with $E^{\prime} \subseteq E$. A cycle (circuit) on $G$ 
is defined as a set of edges that form a closed circuit (cycle). Let $c(G)$ denote the number of linearly independent cycles in $G$. A tree graph is a connected graph that contains no cycles. A spanning tree is a spanning subgraph that is a tree graph.

The chromatic polynomial of $G, P(G, q)$, counts the number of ways of assigning $q$ colors to the vertices of $G$ subject to the condition that no two adjacent vertices have the same color. This has an expression as a sum of contributions from spanning subgraphs $G^{\prime} \subseteq G$ as

$$
P(G, q)=\sum_{G^{\prime} \subseteq G}(-1)^{e\left(G^{\prime}\right)} q^{k\left(G^{\prime}\right)} .
$$

From Eq. (A19), it is clear that $P(G, q)$ always contains a factor of $q$, so one can extract this and define a reduced polynomial

$$
P_{r}(G, q) \equiv \frac{P(G, q)}{q}
$$

The partition function of the $q$-state Potts model, $Z(G, q, v)$, has an expression as a sum of contributions from spanning subgraphs $G^{\prime} \subseteq G$ as [39, 40]

$$
Z(G, q, v)=\sum_{G^{\prime} \subseteq G} v^{e\left(G^{\prime}\right)} q^{k\left(G^{\prime}\right)} .
$$

The chromatic polynomial is a special case of this partition function: $P(G, q)=Z(G, q,-1)$, where $v=-1$ corresponds to the zero-temperature limit of the antiferromagnet. The groundstate degeneracy, per vertex, of the Potts antiferromagnet on a graph $G$ in the limit $n(G) \rightarrow$ $\infty$ is

$$
W(\{G\}, q)=\lim _{n(G) \rightarrow \infty}[P(G, q)]^{1 / n(G)} .
$$

The Tutte polynomial $T(G, x, y)$ is given by

$$
T(G, x, y)=\sum_{G^{\prime} \subseteq G}(x-1)^{k\left(G^{\prime}\right)-k(G)}(y-1)^{c\left(G^{\prime}\right)} .
$$

This is equivalent to the Potts model partition function:

$$
Z(G, q, v)=(x-1)^{k(G)}(y-1)^{n(G)} T(G, x, y)
$$

with the definitions $x=1+(q / v)$ and $y=v+1$. The dimensionless reduced free energy (per vertex) of the Potts model on a graph $G$, in the limit $n(G) \rightarrow \infty$, is

$$
f(\{G\}, q, v)=\lim _{n(G) \rightarrow \infty} \frac{1}{n(G)} \ln [Z(G, q, v)]
$$


The number of spanning trees on a graph $G$, denoted $N_{S T}(G)$, is given by

$$
N_{S T}(G)=T(G, 1,1) .
$$

With $G_{p l}$ a planar graph, one has $N_{S T}\left(G_{p l}\right)=N_{S T}\left(G_{p l}^{*}\right)$. Defining $\tau(\{G\})=$ $\lim _{n(G) \rightarrow \infty}\left[N_{S T}(G)\right]^{1 / n(G)}$ as in the text, we have

$$
\tau\left(G_{p l}^{*}\right)=\left[\tau\left(G_{p l}\right)\right]^{\nu\left(G_{p l}^{*}\right)}=\left[\tau\left(G_{p l}\right)\right]^{1 / \nu\left(G_{p l}\right)}
$$

Hence, for homopolygonal lattices, $\tau(h c)=[\tau(\text { tri })]^{1 / 2}$ and for heteropolygonal lattices,

$$
\begin{gathered}
\tau\left(\Lambda_{\text {dual }}\right)=[\tau(\Lambda)]^{2} \quad \text { for } \Lambda=\left(3 \cdot 12^{2}\right), \quad\left(4 \cdot 8^{2}\right), \quad(4 \cdot 6 \cdot 12) \\
\left.\tau\left(\Lambda_{\text {dual }}\right)\right)=\tau(\Lambda) \quad \text { for } \Lambda=(3 \cdot 6 \cdot 3 \cdot 6), \quad(3 \cdot 4 \cdot 6 \cdot 4)
\end{gathered}
$$

and

$$
\tau\left(\Lambda_{\text {dual }}\right)=[\tau(\Lambda)]^{2 / 3} \quad \text { for } \Lambda=\left(3^{3} \cdot 4^{2}\right), \quad\left(3^{2} \cdot 4 \cdot 3 \cdot 4\right), \quad\left(3^{4} \cdot 6\right) .
$$

Since the values of $\tau(\Lambda)$ are known exactly for all of the Archimedean lattices, these relations yield the values of $\tau\left(\Lambda_{\text {dual }}\right)$ for all of the dual Archimedean lattices. These are listed in Table XXVII,

[1] S.-C. Chang and R. Shrock, Asymptotic behavior of acyclic and totally cyclic orientations of families of directed lattice graphs, arXiv:1810.07357.

[2] For relevant graph theory background, see, e.g., N. Biggs, Algebraic Graph Theory (Cambridge Univ. Press, Cambridge, UK, 1993); D. J. A. Welsh, Complexity: Knots, Colourings, and Counting (Cambridge Univ. Press, Cambridge, UK, 1993); B. Bollobás, Modern Graph Theory (Springer, New York, 1998); and G. Chartrand and L. Lesniak, Graphs and Digraphs (Chapman and Hall/CRC, New York, 2005)

[3] B. Grünbaum and G. C. Shephard, Tilings and Patterns: An Introduction (Freeman, New York, 1989).

[4] For reviews of chromatic polynomials, see, e.g., R. C. Read and W. T. Tutte, "Chromatic Polynomials", in Selected Topics in Graph Theory, 3, eds. L. W. Beineke and R. J. Wilson (Academic Press, New York, 1988), pp. 15-42 and F. M. Dong, K. M. Koh, and K. L. Teo, Chromatic Polynomials and Chromaticity of Graphs (World Scientific, Singapore, 2005). 
[5] R. P. Stanley, Acyclic orientations of graphs, Discrete Math. 5, 171-178 (1973).

[6] C. Greene and T. Zaslavsky, On the interpretation of Whitney numbers through arrangements of hyperplanes, zonotopes, non-Radon partitions, and orientations of graphs, Trans. Amer. Math. Soc. 280, 97-126 (1983).

[7] W. T. Tutte, A contribution to the theory of chromatic polynomials, Canadian J. Math. 6, 80-91 (1954).

[8] W. T. Tutte, On dichromatic polynomials, J. Combin. Theory 2, 301-320 (1967).

[9] T. Brylawski and J. Oxley, The Tutte polynomial and its applications, in N. White, ed., Matroid Applications, vol. 40 of Encyclopedia of Mathematics and its Applications (Cambridge Univ. Press, Cambridge, UK, 1992), pp. 123-225.

[10] D. J. A. Welsh and C. Merino, The Potts model and the Tutte polynomial, J. Math. Phys., 41, 1127-1152 (2000).

[11] D. D. Gebhard and B. E. Sagan, Sinks in acyclic orientations of graphs, J. Combin. Theory B 80, 130-146 (2000).

[12] C. Merino and D. J. A. Welsh, Forest, colorings, and acyclic orientations of the square lattice, Ann. Combin. 3, 417-429 (1999).

[13] N. Calkin, C. Merino, S. Noble and M. Noy, Improved bounds for the number of forests and acyclic orientations in the square lattice, Electron. J. Combin. 10 (R4), 1-18 (2003).

[14] S.-C. Chang and R. Shrock, Tutte polynomials and related asymptotic limiting functions for recursive families of graphs (talk given by R. Shrock at Workshop on Tutte polynomials, Centre de Recerca Matemática (CRM), Sept. 2001, Univ. Autonoma de Barcelona), Adv. Appl. Math. 32, 44-87 (2004).

[15] M. Las Vergnas, Acyclic and totally cyclic orientations of combinatorial geometries, Discrete Math. 20, 51-61 (1977).

[16] M. Las Vergnas, Convexity in oriented matroids, J. Combin. Theory B 29, 231-243 (1980).

[17] N. L. Biggs, R. M. Damerell, and D. A. Sands, Recursive families of graphs, J. Combin. Theory B 12, 123-131 (1972).

[18] R. Shrock and S.-H. Tsai, Lower bounds and series for the ground state entropy of the Potts 
antiferromagnet on Archimedean lattices and their duals, Phys. Rev. E 56, 4111-4124 (1997).

[19] M. Roček, R. Shrock, and S.-H. Tsai, Chromatic polynomials for families of strip graphs and their asymptotic limits, Physica A 252, 505-546 (1998).

[20] R. Shrock and S.-H. Tsai, "Ground state degeneracy of Potts antiferromagnets on 2D lattices: approach using infinite cyclic strip graphs, Phys. Rev. E60, 3512-3515 (1999).

[21] R. Shrock and S.-H. Tsai, Exact partition functions for Potts antiferromagnets on cyclic lattice strips, Physica A 275, 429-449 (2000).

[22] S.-C. Chang and W. Wang, Spanning trees on lattices and integral identities, J. Phys. A 39, 10263-10275 (2006).

[23] R. Shrock, Chromatic polynomials and their zeros and asymptotic limits for families of graphs, Discrete Math. 231, 421-446 (2001).

[24] R. Shrock and S.-H. Tsai, Asymptotic limits and zeros of chromatic polynomials and ground state entropy of Potts antiferromagnets, Phys. Rev. E 55, 5165-5179 (1997).

[25] R. Shrock and S.-H. Tsai, Upper and lower bounds for the ground state entropy of antiferromagnetic Potts models, Phys. Rev. E 55, 6791-6794 (1997).

[26] R. Shrock and S.-H. Tsai, Ground state entropy of antiferromagnetic Potts models: Bounds, series, and Monte Carlo measurements, Phys. Rev. E 56, 2733-2737 (1997).

[27] S.-C. Chang and R. Shrock, Improved lower bounds on ground state entropy of the antiferromagnetic Potts model, Phys. Rev. E 91, 052142 (2015).

[28] N. L. Biggs, Colouring square lattice graphs, Bull. London Math. Soc. 9, 54-56 (1977).

[29] F. Y. Wu, Number of spanning trees on a lattice, J. Phys. A 10, L113-L115 (1977).

[30] R. Shrock and F. Y. Wu, Spanning trees on graphs and lattices in $d$ dimensions, J. Phys. A, 33, 3881-3902 (2000).

[31] S.-C. Chang and R. Shrock, Some exact results for spanning trees on lattices, J. Phys. A 39, 5653-5658 (2006).

[32] R. J. Baxter, Chromatic polynomials of large triangular lattices, J. Phys. A 20, 5241-5261 (1987).

[33] C. Thomassen, Spanning trees and orientations of graphs, J. Combin. 1, 101-111 (2010). 
[34] R. Conde and C. Merino, Comparing the number of acyclic and totally cyclic orientations with that of spanning trees of a graph, Int. J. Math. Combin. 2, 79-89 (2009).

[35] C. Merino, M. Ibañez, and M. Guadalupe Rodrígez, A note on some inequalities for the Tutte polynomial of a matroid, Electronic Notes in Direcrete Math. 34, 603-607 (2009).

[36] L. E. Chávez-Lomeli, C. Merino, S. D. Noble, and M. Ramírez-Ibáñez, Some inequalities for the Tutte polynomial, Eur. J. Combin. 32, 422-433 (2011).

[37] S. D. Noble and G. F. Royle, The Merino-Welsh conjecture holds for series-parallel graphs, Eur. J. Combin. 38, 24-35 (2014).

[38] K. Knauer, L. Martínez-Sandoval, and J. Luis Ramírez-Alfonsín, A Tutte polynomial inequality for lattice path matroids, arXiv:1510.00600.

[39] F. Y. Wu, The Potts model, Rev. Mod. Phys. 54, 235-268 (1982).

[40] C. M. Fortuin and P. W. Kasteleyn, On the random cluster model, Physica 57, 536-564 (1972). 
TABLE I: Lower bounds and their ratios for $\alpha\left(\left(4 \cdot 8^{2}\right)\right)$ as functions of strip width $L_{y}$. In this table and the others, the abbreviation cyl stands for "cylindrical".

\begin{tabular}{||l|l|l|l||}
\hline $\mathrm{BC}$ & $L_{y}$ & {$\left[\lambda_{\left(4 \cdot 8^{2}\right), L_{y}, \text { free } / \text { cyl }}(-1)\right]^{1 /\left(4 L_{y}\right)}$} & $R_{\left(4 \cdot 8^{2}\right), \frac{L_{y}+1}{L_{y}} / \frac{L_{y}+2}{L_{y}}, \text { free } / \text { cyl }}(-1)$ \\
\hline \hline free & 2 & $(889)^{1 / 8}=2.33675252$ & \\
\hline free & 3 & 2.461131465 & 1.05322726 \\
\hline free & 4 & 2.52577995 & 1.02626779 \\
\hline free & 5 & 2.56538118 & 1.01567881 \\
\hline free & 6 & 2.592126335 & 1.01042541 \\
\hline \hline cyl & 2 & $\sqrt{7}=2.64575131$ & \\
\hline cyl & 4 & 2.725822615 & 1.03026411 \\
\hline cyl & 6 & 2.7297041765 & 1.001423996 \\
\hline
\end{tabular}

TABLE II: Upper bounds and their ratios for $\alpha\left(\left(4 \cdot 8^{2}\right)\right)$ as functions of strip width $L_{y}$.

\begin{tabular}{||l|l|l|}
\hline$\left(L_{y}+1\right) / L_{y}$ & {$\left[\lambda_{\left(4 \cdot 8^{2}\right), L_{y}+1, \text { free }}(-1) / \lambda_{\left(4 \cdot 8^{2}\right), L_{y}, \text { free }}(-1)\right]^{1 / 4}$} & $R_{\left(4 \cdot 8^{2}\right), \frac{L_{y}^{2}}{\left(L_{y}-1\right)\left(L_{y}+1\right)}, \text { free }}(-1)$ \\
\hline \hline $2 / 1$ & $\frac{(889)^{1 / 4}}{2}=2.73020617$ & \\
\hline $3 / 2$ & 2.73010279 & 1.00003787 \\
\hline $4 / 3$ & 2.73009404 & 1.00000320 \\
\hline $5 / 4$ & 2.73009323 & 1.00000030 \\
\hline $6 / 5$ & 2.730093140 & 1.000000032 \\
\hline
\end{tabular}


TABLE III: Lower bounds and their ratios for $\alpha_{0}\left(\left(4 \cdot 8^{2}\right)\right)$ as functions of strip width $L_{y}$.

\begin{tabular}{||l|l|l|l||}
\hline BC & $L_{y}$ & {$\left[\lambda_{\left(4 \cdot 8^{2}\right), L_{y}, \text { free } / \text { cyl }}(0)\right]^{1 /\left(4 L_{y}\right)}$} & $R_{\left(4 \cdot 8^{2}\right), \frac{L_{y}+1}{L_{y}} / \frac{L_{y}+2}{L_{y}}, \text { free } / \text { cyl }}(0)$ \\
\hline \hline free & 2 & $(21)^{1 / 8}=1.46311146$ & \\
\hline free & 3 & 1.65063068 & 1.12816469 \\
\hline free & 4 & 1.75020633 & 1.06032582 \\
\hline free & 5 & 1.81176342 & 1.03517133 \\
\hline free & 6 & 1.85353652 & 1.02305659 \\
\hline \hline cyl & 2 & $\sqrt{3}=1.73205081$ & \\
\hline cyl & 4 & 1.98451595 & 1.14576082 \\
\hline cyl & 6 & 2.032649948 & 1.024254778 \\
\hline
\end{tabular}

TABLE IV: Upper bounds and their ratios for $\alpha_{0}\left(\left(4 \cdot 8^{2}\right)\right)$ as functions of strip width $L_{y}$.

\begin{tabular}{||l|l|l|}
\hline$\left(L_{y}+1\right) / L_{y}$ & {$\left[\lambda_{\left(4 \cdot 8^{2}\right), L_{y}+1, \text { free }}(0) / \lambda_{\left(4 \cdot 8^{2}\right), L_{y}, \text { free }}(0)\right]^{1 / 4}$} & $R_{\left(4 \cdot 8^{2}\right), \frac{L_{y}^{2}}{\left(L_{y}-1\right)\left(L_{y}+1\right)}, f r e e}(0)$ \\
\hline \hline $2 / 1$ & $(21)^{1 / 4}=2.14069514$ & \\
\hline $3 / 2$ & 2.10084938 & 1.01896650 \\
\hline $4 / 3$ & 2.08644655 & 1.00690304 \\
\hline $5 / 4$ & 2.08041720 & 1.00289815 \\
\hline $6 / 5$ & 2.077301063 & 1.001500087 \\
\hline
\end{tabular}


TABLE V: Lower bounds and their ratios for $\beta\left(\left(4 \cdot 8^{2}\right)\right)$ as functions of strip width $L_{y}$. The abbreviation tor stands for "toroidal".

\begin{tabular}{||l|l|l|l||}
\hline $\mathrm{BC}$ & $L_{y}$ & {$\left[\lambda_{\left(4 \cdot 8^{2}\right) L_{y}, \text { cyc } / \text { tor }}(-1,1)\right]^{1 /\left(4 L_{y}\right)}$} & $R_{\left(4 \cdot 8^{2}\right), \frac{L_{y}+1}{L_{y}} / \frac{L_{y}+2}{L_{y}}, \text { cyc } / \text { tor }}(-1,1)$ \\
\hline \hline cyc & 2 & $\sqrt{2}=1.41421356$ & \\
\hline cyc & 3 & 1.62047257 & 1.14584715 \\
\hline cyc & 4 & 1.73110235 & 1.06827007 \\
\hline cyc & 5 & 1.80061384 & 1.04015446 \\
\hline \hline tor & 2 & 2 & \\
\hline tor & 4 & 2.080338691 & 1.040169345 \\
\hline
\end{tabular}

TABLE VI: Upper bounds and their ratios for $\beta\left(\left(4 \cdot 8^{2}\right)\right)$ as functions of strip width $L_{y}$.

\begin{tabular}{||l|l|l|}
\hline$\left(L_{y}+1\right) / L_{y}$ & {$\left[\frac{\lambda_{\left(4 \cdot 8^{2}\right), L_{y}+1, c y c}(-1,1)}{\lambda_{\left(4 \cdot 8^{2}\right), L_{y}, c y c}(-1,1)}\right]^{1 / 4}$} & $R_{\left(4 \cdot 8^{2}\right), \frac{L_{y}^{2}}{\left(L_{y}-1\right)\left(L_{y}+1\right)}, c y c}(-1,1)$ \\
\hline \hline $3 / 2$ & 2.12762488 & \\
\hline $4 / 3$ & 2.11040559 & 1.00815923 \\
\hline $5 / 4$ & 2.107715225 & 1.001276438 \\
\hline
\end{tabular}


TABLE VII: Lower bounds and their ratios for $\alpha(k a g)$ as functions of strip width $L_{y}$. In this table and the others, the abbreviation cyl stands for "cylindrical".

\begin{tabular}{||l|l|l|l||}
\hline BC & $L_{y}$ & {$\left[\lambda_{\text {kag }, L_{y}, \text { free } / \text { cyl }}(-1)\right]^{1 /\left(3 L_{y}\right)}$} & $R_{\text {kag },\left(L_{y}+1\right) / L_{y}, \text { free } / \text { cyl }}(-1)$ \\
\hline \hline free & 1 & $(12)^{1 / 3}=2.289428485$ & \\
\hline free & 2 & $3^{1 / 3}\left(\frac{47+\sqrt{2113}}{2}\right)^{1 / 6}=2.73478917$ & 1.172619975 \\
\hline free & 3 & 2.9014136165 & 1.07624544 \\
\hline \hline cyl & 1 & $(18)^{1 / 3}=2.62074139$ & \\
\hline cyl & 2 & {$[6(88+\sqrt{7609})]^{1 / 6}=3.18879387$} & 1.216752585 \\
\hline cyl & 3 & 3.2490590695 & 1.018899059 \\
\hline
\end{tabular}

TABLE VIII: Upper bounds and their ratios for $\alpha(k a g)$ as functions of strip width $L_{y}$.

\begin{tabular}{||l|l|l||}
\hline$\left(L_{y}+1\right) / L_{y}$ & $\left(\lambda_{k a g, L_{y}+1, \text { free }}(-1) / \lambda_{\text {kag }, L_{y}, \text { free }}(-1)\right)^{1 / 3}$ & $R_{k a g, \frac{L_{y}^{2}}{\left(L_{y}-1\right)\left(L_{y}+1\right)}, \text { free }}(-1)$ \\
\hline \hline $2 / 1$ & {$\left[\frac{3(47+\sqrt{2113})}{8}\right]^{1 / 3}=3.26678550$} & \\
\hline $3 / 2$ & 3.2657371991 & 1.000321000 \\
\hline
\end{tabular}

TABLE IX: Lower bounds on $\alpha_{0}(k a g)$ and their ratios, as functions of strip width $L_{y}$.

\begin{tabular}{||l|l|l|l||}
\hline $\mathrm{BC}$ & $L_{y}$ & {$\left[\lambda_{\text {kag }, L_{y}, \text { free } / \text { cyl }}(0)\right]^{1 /\left(3 L_{y}\right)}$} & $R_{\text {kag },\left(L_{y}+1\right) / L_{y}, \text { free } / \text { cyl }}(0)$ \\
\hline \hline free & 1 & $2^{1 / 3}=1.25992105$ & \\
\hline free & 2 & $2^{1 / 3}(5+\sqrt{23})^{1 / 6}=1.84296413$ & 1.43903358 \\
\hline free & 3 & 2.07555502 & 1.15009316 \\
\hline \hline cyl & 1 & $2^{2 / 3}=1.58740105$. & \\
\hline cyl & 2 & {$[2(3+\sqrt{11})]^{1 / 3}=2.32901182$} & 1.46718551 \\
\hline cyl & 3 & 2.481974714 & 1.065677167 \\
\hline
\end{tabular}


TABLE X: Upper bounds on $\alpha_{0}(k a g)$ and their ratios, as functions of strip width $L_{y}$.

\begin{tabular}{||l|l|l||}
\hline$\left(L_{y}+1\right) / L_{y}$ & {$\left[\lambda_{\text {kag }, L_{y}+1, \text { free }}(0) / \lambda_{\text {kag }, L_{y}, \text { free }}(0)\right]^{1 / 3}$} & $R_{k a g, \frac{L_{y}^{2}}{\left(L_{y}-1\right)\left(L_{y}+1\right)}, \text { free }}(0)$ \\
\hline \hline $2 / 1$ & {$[2(5+\sqrt{23})]^{1 / 3}=2.695817165$} & \\
\hline $3 / 2$ & 2.632503652 & 1.024050684 \\
\hline
\end{tabular}

TABLE XI: Lower bounds and their ratios for $\beta(k a g)$ as functions of strip width $L_{y}$.

\begin{tabular}{||l|l|l|l||}
\hline BC & $L_{y}$ & {$\left[\lambda_{\text {kag }, L_{y}, \text { cyc }}(-1,1)\right]^{1 /\left(3 L_{y}-1\right)}$ or $\left[\lambda_{\text {kag }, L_{y}, \text { tor }}(-1,1)\right]^{1 /\left(3 L_{y}\right)}$} & $R_{k a g, \frac{L_{y}+1}{L_{y}}, \text { cyc } / \text { tor }}(-1,1)$ \\
\hline \hline cyc & 2 & $(55)^{1 / 5}=2.22880738$ & \\
\hline cyc & 3 & 2.653725025 & 1.19064799 \\
\hline \hline tor & 1 & $(30)^{1 / 3}=3.10723251$ & \\
\hline tor & 2 & {$[10((79+2 \sqrt{1585})])^{1 / 6}=3.415032724$} & 1.099059281 \\
\hline
\end{tabular}

TABLE XII: Upper bounds and their ratios for $\beta(k a g)$ as functions of strip width $L_{y}$.

\begin{tabular}{||l|l|l|}
\hline$\left(L_{y}+1\right) / L_{y}$ & {$\left[\frac{\lambda_{k a g, L_{y}+1, c y c}(-1,1)}{\lambda_{k a g, L_{y}, c y c}(-1,1)}\right]^{1 / 3}$} & $R_{k a g, \frac{L_{y}^{2}}{\left(L_{y}-1\right)\left(L_{y}+1\right)}, c y c}(-1,1)$ \\
\hline \hline $2 / 1$ & $(55)^{1 / 3}=3.80295246$ & \\
\hline $3 / 2$ & 3.549454037 & 1.071418990. \\
\hline
\end{tabular}


TABLE XIII: Lower bounds on $\alpha\left(\left(3^{3} \cdot 4^{2}\right)\right)$ and their ratios, as functions of strip width $L_{y}$.

\begin{tabular}{||l|l|l|l||}
\hline BC & $L_{y}$ & {$\left[\lambda_{\left(3^{3} \cdot 4^{2}\right), L_{y}, \text { free } / \text { cyl }}(-1)\right]^{1 /\left(2 L_{y}\right)}$} & $R_{\left(3^{3} .4^{2}\right), \frac{L_{y}+2}{L_{y}} / \frac{L_{y}+1}{L_{y}}, \text { free } / \text { cyl }}(-1)$ \\
\hline \hline free & 3 & $\begin{array}{l}(17+4 \sqrt{13})^{1 / 3} \\
=3.15557776\end{array}$ & \\
\hline free & 5 & 3.45440528 & 1.09469820 \\
\hline \hline cyl & 2 & 3 & \\
\hline cyl & 4 & 3.82776685 & 1.27592228 \\
\hline cyl & 6 & 3.922582062 & 1.024770372 \\
\hline \hline free & 2 & $\sqrt{3} \times 7^{1 / 4}=2.81731325$ & \\
\hline free & 3 & $(497+\sqrt{240313})^{1 / 6}$ & 1.12004032 \\
\hline free & 4 & 3.33914866 & 1.05819806 \\
\hline free & 5 & 3.45434518 & 1.03449877 \\
\hline free & 6 & 3.53332068 & 1.02286265 \\
\hline \hline cyl & 2 & $(4 \times 21)^{1 / 4}=3.0274001$ & \\
\hline cyl & 3 & $(2414)^{1 / 6}=3.66260045$ & 1.2098171 \\
\hline cyl & 4 & {$[6(3909+13 \sqrt{89841})]^{1 / 8}$} & 1.04720046 \\
\hline cyl & 5 & $(407837+5 \sqrt{6475806457})^{1 / 10}$ & 1.016341829 \\
\hline
\end{tabular}


TABLE XIV: Upper bounds on $\alpha\left(\left(3^{3} \cdot 4^{2}\right)\right)$ and their ratios, as functions of strip width $L_{y}$.

\begin{tabular}{||l|l|l|}
\hline$\frac{L_{y}+2}{L_{y}}$ or $\frac{L_{y}+1}{L_{y}}$ & $\sqrt{\frac{\lambda_{\left(3^{3} \cdot 4^{2}\right), L_{y}+2 / 1, \text { free }}(-1)}{\lambda_{\left(3^{3} \cdot 4^{2}\right), L_{y}, \text { free }}(-1)}}$ & $R_{\left(3^{3} \cdot 4^{2}\right), \frac{L_{y}^{2}}{\left(L_{y}-2\right)\left(L_{y}+2\right)} / \frac{L_{y}^{2}}{\left(L_{y}-1\right)\left(L_{y}+1\right)}, f r e e}(-1)$ \\
\hline \hline $3 / 1$ & $\frac{2+\sqrt{13}}{\sqrt{2}}=3.96372332$ & \\
\hline $5 / 3$ & 3.95653392 & 1.001817095 \\
\hline \hline $2 / 1$ & $\frac{\sqrt{63}}{2}=3.96862697$ & \\
\hline $3 / 2$ & $\frac{1}{3} \sqrt{71+\frac{\sqrt{240313}}{7}}$ & 1.00254569 \\
$=3.9585497$ & \\
\hline $4 / 3$ & 3.95673204 & 1.00045939 \\
\hline $5 / 4$ & 3.95626750 & 1.00011742 \\
\hline $6 / 5$ & 3.956121920 & 1.000036798 \\
\hline
\end{tabular}


TABLE XV: Lower bounds on $\alpha_{0}\left(\left(3^{3} \cdot 4^{2}\right)\right)$ and their ratios, as functions of strip width $L_{y}$.

\begin{tabular}{||l|l|l|l||}
\hline BC & $L_{y}$ & {$\left[\lambda_{\left(3^{3} \cdot 4^{2}\right), L_{y}, f r e e / c y l}(0)\right]^{1 /\left(2 L_{y}\right)}$} & $R_{\left(3^{3} \cdot 4^{2}\right), \frac{L_{y}+2}{L_{y}} / \frac{L_{y}+1}{L_{y}}, \text { free } / \text { cyl }}(0)$ \\
\hline \hline free & 3 & $\left(\frac{13+\sqrt{105}}{2}\right)^{1 / 3}=2.2652284$ & \\
\hline free & 5 & 2.63777102 & 1.16446139 \\
\hline \hline cyl & 2 & 2 & \\
\hline cyl & 4 & 2.95888008 & 1.47944004 \\
\hline cyl & 6 & 3.142411228 & 1.062027234 \\
\hline \hline free & 2 & $(4 \times 3)^{1 / 4}=1.86120972$ & \\
\hline free & 3 & $\left(\frac{137+\sqrt{17713}}{2}\right)^{1 / 6}=2.26506049$ & 1.21698295 \\
\hline free & 4 & 2.49249353 & 1.10040926 \\
\hline free & 5 & 2.63744871 & 1.05815669 \\
\hline free & 6 & 2.73767800 & 1.03800237 \\
\hline \hline cyl & 2 & $(9 \times 2)^{1 / 4}=2.05976714$ & \\
\hline cyl & 3 & $2^{5 / 6} \times 13^{1 / 6}=2.73221930$ & 1.32646999 \\
\hline cyl & 4 & $(3096+6 \sqrt{264981})^{1 / 8}$ & 1.08992900 \\
\hline cyl & 5 & $(39973+\sqrt{1566836161})^{1 / 10}$ & 1.037895636 \\
\hline
\end{tabular}


TABLE XVI: Upper bounds on $\alpha_{0}\left(\left(3^{3} \cdot 4^{2}\right)\right)$ and their ratios, as functions of strip width $L_{y}$.

\begin{tabular}{||l|l|l|}
\hline$\frac{L_{y}+2}{L_{y}}$ or $\frac{L_{y}+1}{L_{y}}$ & $\sqrt{\frac{\lambda_{\left(3^{3} \cdot 4^{2}\right), L_{y}+2 / 1, \text { free }}(0)}{\lambda_{\left(3^{3} \cdot 4^{2}\right), L_{y}, \text { free }}(0)}}$ & $R_{\left(3^{3} \cdot 4^{2}\right), \frac{L_{y}^{2}}{\left(L_{y}-2\right)\left(L_{y}+2\right)} / \frac{L_{y}^{2}}{\left(L_{y}-1\right)\left(L_{y}+1\right)}, \text { free }}(0)$ \\
\hline \hline $3 / 1$ & $\frac{\sqrt{21}+\sqrt{5}}{2}=3.40932184$ & \\
\hline $5 / 3$ & 3.31455119 & 1.0285923 \\
\hline \hline $2 / 1$ & $2 \sqrt{3}=3.464101615$ & \\
\hline $3 / 2$ & $\frac{1}{2} \sqrt{\frac{137+\sqrt{17713}}{6}}$ & 1.03262310 \\
$=3.35466215$ & \\
\hline $5 / 3$ & 3.32121311 & 1.01007133 \\
\hline $6 / 5$ & 3.30661746 & 1.00441407 \\
\hline
\end{tabular}

TABLE XVII: Lower bounds and their ratios for $\beta\left(3^{3} \cdot 4^{2}\right)$ as functions of strip width $L_{y}$.

\begin{tabular}{||l|l|l|l||}
\hline BC & $L_{y}$ & $\lambda_{\left(3^{3} \cdot 4^{2}\right), L_{y}, \text { cyc/tor }}^{1 /\left(2 L_{y}\right)}(-1,1)$ & $R_{\left(3^{3} \cdot 4^{2}\right), \frac{L_{y}+2}{L_{y}} / \frac{L_{y}+1}{L_{y}}, \text { cyc/tor }}(-1,1)$ \\
\hline \hline cyc & 3 & 3.32494691 & \\
\hline cyc & 5 & 4.03289325 & 1.21291959 \\
\hline \hline tor & 2 & $\frac{\sqrt{2(25+\sqrt{613})}}{2}=4.987927265$ & \\
\hline tor & 4 & 5.26288016 & 1.05512368 \\
\hline \hline cyc & 2 & $(43)^{1 / 4}=2.5607496$ & \\
\hline cyc & 3 & 3.31487994 & 1.2944959 \\
\hline cyc & 4 & 3.74829168 & 1.1307473 \\
\hline cyc & 5 & 4.02663152 & 1.0742578 \\
\hline \hline tor & 2 & $(584)^{1 / 4}=4.91590195$ & \\
\hline tor & 3 & 5.176853205 & 1.05308309 \\
\hline
\end{tabular}


TABLE XVIII: Upper bounds and their ratios for $\beta\left(3^{3} \cdot 4^{2}\right)$ as functions of strip width $L_{y}$.

\begin{tabular}{||l|l|l|}
\hline$\frac{L_{y}+2}{L_{y}}$ or $\frac{L_{y}+1}{L_{y}}$ & $\sqrt{\frac{\lambda_{\left(3^{3} \cdot 4^{2}\right), L_{y}+2 / 1, c y c}(-1,1)}{\lambda_{\left(3^{3} \cdot 4^{2}\right), L_{y}, c y c}(-1,1)}}$ & $R_{\left(3^{3} \cdot 4^{2}\right), \frac{L_{y}^{2}}{\left(L_{y}-2\right)\left(L_{y}+2\right)} / \frac{L_{y}^{2}}{\left(L_{y}-1\right)\left(L_{y}+1\right)}, c y c}(-1,1)$ \\
\hline \hline $3 / 1$ & 6.06285349 & \\
\hline $5 / 3$ & 5.38722039 & 1.12541404 \\
\hline \hline $2 / 1$ & $\sqrt{43}=6.5574385$ & \\
\hline $3 / 2$ & 5.55480969 & 1.18049742 \\
\hline $4 / 3$ & 5.41913669 & 1.02503591 \\
\hline $5 / 4$ & 5.362606470 & 1.010541556 \\
\hline
\end{tabular}

TABLE XIX: Lower bounds on $\alpha\left(\left(3^{2} \cdot 4 \cdot 3 \cdot 4\right)\right)$ and their ratios, as functions of strip width $L_{y}$.

\begin{tabular}{||l|l|l|l||}
\hline BC & $L_{y}$ & {$\left[\lambda_{\left(3^{2} \cdot 4 \cdot 3 \cdot 4\right), L_{y}, \text { free } / \text { cyl }}(-1)\right]^{1 /\left(2 L_{y}\right)}$} & $R_{\left(3^{2} \cdot 4 \cdot 3 \cdot 4\right), \frac{L_{y}+1}{L_{y}} / \frac{L_{y}+2}{L_{y}}, \text { free } / \text { cyl }}(-1)$ \\
\hline \hline free & 2 & $(9 \times 7)^{1 / 4}=2.81731325$ & \\
\hline free & 3 & $\begin{array}{l}(17+4 \sqrt{13})^{1 / 3} \\
=3.15557776\end{array}$ & 1.12006635 \\
\hline free & 4 & 3.33926081 & 1.05820901 \\
\hline free & 5 & 3.45448103 & 1.03450471 \\
\hline free & 6 & 3.53347262 & 1.02286641 \\
\hline \hline cyl & 2 & 3 & \\
\hline cyl & 4 & 3.82776685 & 1.27592228 \\
\hline cyl & 6 & 3.922582062 & 1.024770372 \\
\hline
\end{tabular}


TABLE XX: Upper bounds on $\alpha\left(\left(3^{2} \cdot 4 \cdot 3 \cdot 4\right)\right)$ and their ratios, as functions of strip width $L_{y}$.

\begin{tabular}{||l|l|l||}
\hline$\left(L_{y}+1\right) / L_{y}$ & $\sqrt{\frac{\lambda_{\left(3^{2} \cdot 4 \cdot 3 \cdot 4\right), L_{y}+1, \text { free }}(-1)}{\lambda_{\left(3^{2} \cdot 4 \cdot 3 \cdot 4\right), L_{y}, \text { free }}(-1)}}$ & $R_{\left(3^{2} \cdot 4 \cdot 3 \cdot 4\right), \frac{L_{y}^{2}}{\left(L_{y}-1\right)\left(L_{y}+1\right)}, \text { free }}(-1)$ \\
\hline \hline $2 / 1$ & $\frac{3 \sqrt{7}}{2}=3.96862697$ & \\
\hline $3 / 2$ & $\frac{17+4 \sqrt{13}}{3 \sqrt{7}}=3.9588257$ & 1.00247579 \\
\hline $4 / 3$ & 3.95698775 & 1.00046449 \\
\hline $5 / 4$ & 3.95651388 & 1.00011977 \\
\hline $6 / 5$ & 3.956364741 & 1.000037697 \\
\hline
\end{tabular}

TABLE XXI: Lower bounds on $\alpha_{0}\left(\left(3^{2} \cdot 4 \cdot 3 \cdot 4\right)\right)$ and their ratios, as functions of strip width $L_{y}$.

\begin{tabular}{||l|l|l|l||}
\hline BC & $L_{y}$ & {$\left[\lambda_{\left(3^{2} \cdot 4 \cdot 3 \cdot 4\right), L_{y}, \text { free } / \text { cyl }}(0)\right]^{1 /\left(2 L_{y}\right)}$} & $R_{\left(3^{2} \cdot 4 \cdot 3 \cdot 4\right), \frac{L_{y}+1}{L_{y}} / \frac{L_{y}+2}{L_{y}}, \text { free } / \text { cyl }}(0)$ \\
\hline \hline free & 2 & $(4 \times 3)^{1 / 4}=1.86120972$ & \\
\hline free & 3 & $\left(\frac{13+\sqrt{105}}{2}\right)^{1 / 3}=2.26522841$ & 1.21707317 \\
\hline free & 4 & 2.49270953 & 1.10042304 \\
\hline free & 5 & 2.63768181 & 1.05815851 \\
\hline free & 6 & 2.73791775 & 1.03800153 \\
\hline \hline cyl & 2 & 2 & \\
\hline cyl & 4 & 2.95888008 & 1.47944004 \\
\hline cyl & 6 & 3.142411229 & 1.062027234 \\
\hline
\end{tabular}


TABLE XXII: Upper bounds on $\alpha_{0}\left(n\left(3^{2} \cdot 4 \cdot 3 \cdot 4\right)\right)$ and their ratios, as functions of strip width $L_{y}$.

\begin{tabular}{||l|l|l||}
\hline$\left(L_{y}+1\right) / L_{y}$ & $\sqrt{\frac{\lambda_{\left(3^{2} \cdot 4 \cdot 3 \cdot 4\right), L_{y}+1, \text { free }}(0)}{\lambda_{\left(3^{2} \cdot 4 \cdot 3 \cdot 4\right), L_{y}, \text { free }}(0)}}$ & $R_{\left(3^{2} \cdot 4 \cdot 3 \cdot 4\right), \frac{L_{y}^{2}}{\left(L_{y}-1\right)\left(L_{y}+1\right)}, \text { free }}(0)$ \\
\hline \hline $2 / 1$ & $2 \sqrt{3}=3.464101615$ & \\
\hline $3 / 2$ & $\frac{13+\sqrt{105}}{4 \sqrt{3}}=3.35540832$ & 1.03239346 \\
\hline $4 / 3$ & 3.32162574 & 1.01017050 \\
\hline $5 / 4$ & 3.30693243 & 1.00444318 \\
\hline $6 / 5$ & 3.299213098 & 1.002339750 \\
\hline
\end{tabular}

TABLE XXIII: Lower bounds and their ratios for $\beta\left(3^{2} \cdot 4 \cdot 3 \cdot 4\right)$ as functions of strip width $L_{y}$.

\begin{tabular}{||l|l|l|l||}
\hline $\mathrm{BC}$ & $L_{y}$ & {$\left[\lambda_{\left(3^{2} \cdot 4 \cdot 3 \cdot 4\right), L_{y}, \text { cyc/tor }}(-1,1)\right]^{1 /\left(2 L_{y}\right)}$} & $R_{\left(3^{2} \cdot 4 \cdot 3 \cdot 4\right), \frac{L_{y}+1}{L_{y}} / \frac{L_{y}+2}{L_{y}}, \text { cyc } / \text { tor }}(-1,1)$ \\
\hline \hline cyc & 2 & $(43)^{1 / 4}=2.56074960$ & \\
\hline cyc & 3 & 3.32202041 & 1.29728436 \\
\hline cyc & 4 & 3.75433393 & 1.13013572 \\
\hline cyc & 5 & 4.03143679 & 1.07380879 \\
\hline \hline tor & 2 & $\frac{\sqrt{2(25+\sqrt{613})}}{2}=4.987927265$ & \\
\hline tor & 4 & 5.264056522 & 1.055359520 \\
\hline
\end{tabular}

TABLE XXIV: Upper bounds and their ratios for $\beta\left(3^{2} \cdot 4 \cdot 3 \cdot 4\right)$ as functions of strip width $L_{y}$.

\begin{tabular}{||l|l|l|}
\hline$\frac{L_{y}+1}{L_{y}}$ & $\sqrt{\frac{\lambda_{\left(3^{2} \cdot 4 \cdot 3 \cdot 4\right), L_{y}+1, c y c}(-1,1)}{\lambda_{\left(3^{2} \cdot 4 \cdot 3 \cdot 4\right), L_{y}, c y c}(-1,1)}}$ & $R_{\left(3^{2} \cdot 4 \cdot 3 \cdot 4\right), \frac{L_{y}^{2}}{\left(L_{y}-1\right)\left(L_{y}+1\right)}, c y c}(-1,1)$ \\
\hline \hline $2 / 1$ & $\sqrt{43}=6.55743852$ & \\
\hline $3 / 2$ & 5.59078335 & 1.17290156 \\
\hline $4 / 3$ & 5.41906930 & 1.03168700 \\
\hline $5 / 4$ & 5.360035653 & 1.011013668 \\
\hline
\end{tabular}


TABLE XXV: Values of the exponential growth constants (EGCs) $\alpha(\Lambda), \alpha_{0}(\Lambda)$, and $\beta(\Lambda)$ for the Archimedean lattices $\Lambda$ analyzed here via calculations on sequences of finite-width, infinite-length strips. In the right-most column we list the exactly known values of $\tau(\Lambda)$. The lattices are listed in order of increasing vertex degree $\Delta(\Lambda)$ and, for a given vertex degree, in order of increasing girth, $g(\Lambda)$. For the EGCs that are not exactly known, we list the approximate values that we have obtained from our upper and lower bounds, as defined in Eq. (4.10). In the case of the homopolygonal lattices, (hc), (sq), and (tri), we list the exact values of $\left(\alpha(t r i), \alpha_{0}(\operatorname{tri})\right.$, and $\beta(h c)$ and the approximate values of the other EGCs that we obtained in [1]. See text for further discussion.

\begin{tabular}{|c|c|c|c|c|c|c|}
\hline \hline$\Lambda$ & $\Delta(\Lambda)$ & $g(\Lambda)$ & $\alpha(\Lambda)$ & $\alpha_{0}(\Lambda)$ & $\beta(\Lambda)$ & $\tau(\Lambda)$ \\
\hline$\left(4 \cdot 8^{2}\right)$ & 3 & 4 & $2.7299 \pm 0.0002$ & $2.055 \pm 0.022$ & $2.094 \pm 0.014$ & 2.196103 \\
$\left(6^{3}\right)=\mathrm{hc}$ & 3 & 6 & $2.78284 \pm 0.00064$ & $2.134 \pm 0.027$ & 2.115336 & 2.242665 \\
\hline$(3 \cdot 6 \cdot 3 \cdot 6)$ & 4 & 3 & $3.2574 \pm 0.0083$ & $2.557 \pm 0.075$ & $3.482 \pm 0.067$ & 3.113341 \\
$\left(4^{4}\right)=\mathrm{sq}$ & 4 & 4 & $3.49359 \pm 0.00034$ & $2.846 \pm 0.016$ & $3.49359 \pm 0.00034$ & 3.209912 \\
\hline$\left(3^{3} \cdot 4^{2}\right)$ & 5 & 3 & $3.939 \pm 0.017$ & $3.221 \pm 0.078$ & $5.313 \pm 0.050$ & 4.083383 \\
$\left(3^{2} \cdot 4 \cdot 3 \cdot 4\right)$ & 5 & 3 & $3.939 \pm 0.017$ & $3.221 \pm 0.078$ & $5.312 \pm 0.048$ & 4.099462 \\
\hline$\left(3^{6}\right)=\operatorname{tri}$ & 6 & 3 & 4.474647 & 3.770920 & $7.7442 \pm 0.0036$ & 5.029546 \\
\hline \hline
\end{tabular}


TABLE XXVI: Values of $\alpha_{u, w}(\Lambda), \alpha_{0, u, w}(\Lambda)$, and $\beta_{u, w}(\Lambda)$ or $\beta_{u, w^{\prime}}(\Lambda)$ for Archimedean lattices $\Lambda$. The last column lists the (exactly known) values of $\tau(\Lambda)$. See text for definitions and notation.

\begin{tabular}{|c|c|c|c|c|c|c|}
\hline \hline$\Lambda$ & $\Delta(\Lambda)$ & $g(\Lambda)$ & $\alpha_{u, w}(\Lambda)$ & $\alpha_{0, u, w}(\Lambda)$ & $\beta_{u, w}(\Lambda)$ & $\tau(\Lambda)$ \\
\hline$\left(3 \cdot 12^{2}\right)$ & 3 & 3 & 2.569587 & 1.878922 & 2.039649 & 2.055591 \\
$\left(4 \cdot 8^{2}\right)$ & 3 & 4 & 2.730206 & 2.140695 & 2.101400 & 2.196103 \\
$(4 \cdot 6 \cdot 12)$ & 3 & 4 & 2.721014 & 2.097345 & 2.108019 & 2.1766685 \\
$\left(6^{3}\right)=\mathrm{hc}$ & 3 & 6 & 2.783882 & 2.236068 & 2.121320 & 2.242665 \\
\hline$(3 \cdot 6 \cdot 3 \cdot 6)$ & 4 & 3 & 3.267168 & 2.714418 & 3.5 & 3.113341 \\
$(3 \cdot 4 \cdot 6 \cdot 4)$ & 4 & 3 & 3.381580 & 2.853639 & 3.5 & 3.141816 \\
$\left(4^{4}\right)=\mathrm{sq}$ & 4 & 4 & 3.5 & 3 & 3.5 & 3.209912 \\
\hline$\left(3^{3} \cdot 4^{2}\right)$ & 5 & 3 & 3.968627 & 3.464102 & 5.303301 & 4.083383 \\
$\left(3^{2} \cdot 4 \cdot 3 \cdot 4\right)$ & 5 & 3 & 3.968627 & 3.464102 & 5.303301 & 4.099462 \\
$\left(3^{4} \cdot 6\right)$ & 5 & 3 & 3.834352 & 3.295098 & 5.303301 & 4.022983 \\
\hline$(3)^{6}=$ tri & 6 & 3 & 4.5 & 4 & 7.75 & 5.029546 \\
\hline \hline
\end{tabular}


TABLE XXVII: Values of $\alpha_{u, w}\left(\Lambda_{d u a l}\right), \alpha_{0, u, w}\left(\Lambda_{d u a l}\right)$, and $\beta_{u, w}\left(\Lambda_{d u a l}\right)$ for duals of Archimedean lattices, $\Lambda_{d u a l}$. For the $\left[3 \cdot 12^{2}\right],\left[4 \cdot 8^{2}\right]$, and $[4 \cdot 6 \cdot 12]$ lattices we list the values of $\alpha_{u, w^{\prime}}$ and $\alpha_{0, u, w^{\prime}}$. In the last column we list the (exactly known) values of $\tau\left(\Lambda_{\text {dual }}\right)$. See text for definitions and notation.

\begin{tabular}{|c|c|c|c|c|c|c|}
\hline \hline$\Lambda_{\text {dual }}$ & $p\left(\Lambda_{\text {dual }}\right)$ & $\Delta_{\text {eff }}\left(\Lambda_{\text {dual }}\right)$ & $\alpha_{u, w}\left(\Lambda_{\text {dual }}\right)$ & $\alpha_{0, u, w}\left(\Lambda_{\text {dual }}\right)$ & $\beta_{u, w}\left(\Lambda_{\text {dual }}\right)$ & $\tau\left(\Lambda_{\text {dual }}\right)$ \\
\hline$\left[3 \cdot 12^{2}\right]$ & 3 & 6 & 4.160168 & 3.301927 & 6.6027795 & 4.225454 \\
{$\left[4 \cdot 8^{2}\right]$} & 3 & 6 & 4.415880 & 3.741657 & 7.454026 & 4.822867 \\
{$[4 \cdot 6 \cdot 12]$} & 3 & 6 & 4.443744 & 3.825862 & 7.403934 & 4.737886 \\
{$\left[6^{3}\right]=$ tri } & 3 & 6 & 4.5 & 4 & 7.75 & 5.029546 \\
\hline$[3 \cdot 6 \cdot 3 \cdot 6]$ & 4 & 4 & 3.5 & 3 & 3.2671675 & 3.113341 \\
{$[3 \cdot 4 \cdot 6 \cdot 4]$} & 4 & 4 & 3.5 & 3 & 3.381580 & 3.141816 \\
{$\left[4^{4}\right]=\mathrm{sq}$} & 4 & 4 & 3.5 & 3 & 3.5 & 3.209912 \\
\hline$\left[3^{3} \cdot 4^{2}\right]$ & 5 & $10 / 3$ & 3.041101 & 2.519842 & 2.506649 & 2.554740 \\
{$\left[3^{2} \cdot 4 \cdot 3 \cdot 4\right]$} & 5 & $10 / 3$ & 3.041101 & 2.519842 & 2.506649 & 2.561442 \\
{$\left[3^{4} \cdot 6\right]$} & 5 & $10 / 3$ & 3.041101 & 2.519842 & 2.449785 & 2.529485 \\
\hline$\left[3^{6}\right]=\mathrm{hc}$ & 6 & 3 & 2.783882 & 2.236068 & 2.121320 & 2.242665 \\
\hline \hline
\end{tabular}

